\title{
Closable multipliers
}

\author{
V.S. Shulman, I.G. Todorov and L. Turowska
}

\begin{abstract}
Let $(X, \mu)$ and $(Y, \nu)$ be standard measure spaces. A function $\varphi \in L^{\infty}(X \times Y, \mu \times \nu)$ is called a (measurable) Schur multiplier if the map $S_{\varphi}$, defined on the space of Hilbert-Schmidt operators from $L_{2}(X, \mu)$ to $L_{2}(Y, \nu)$ by multiplying their integral kernels by $\varphi$, is bound-ed in the operator norm.

The paper studies measurable functions $\varphi$ for which $S_{\varphi}$ is closable in the norm topology or in the weak* topology. We obtain a characterisation of $\mathrm{w}^{*}$-closable multipliers and relate the question about norm closability to the theory of operator synthesis. We also study multipliers of two special types: if $\varphi$ is of Toeplitz type, that is, if $\varphi(x, y)=f(x-y)$, $x, y \in G$, where $G$ is a locally compact abelian group, then the closability of $\varphi$ is related to the local inclusion of $f$ in the Fourier algebra $A(G)$ of $G$. If $\varphi$ is a divided difference, that is, a function of the form $(f(x)-f(y)) /(x-y)$, then its closability is related to the "operator smoothness" of the function $f$. A number of examples of non-closable, norm closable and $\mathrm{w}^{*}$-closable multipliers are presented.
\end{abstract}

Mathematics Subject Classification (2010). Primary 47B49; Secondary 47L60, 43A22.

\section{Introduction}

Let $(X, \mu)$ and $(Y, \nu)$ be standard measure spaces, $H_{1}=L^{2}(X, \mu), H_{2}=$ $L^{2}(Y, \nu)$, and let $\mathcal{B}\left(H_{1}, H_{2}\right)$ be the space of all bounded linear operators from $H_{1}$ into $H_{2}$. There is a method, due mainly to Birman and Solomyak $[2,3,4,5]$, to associate to certain bounded measurable functions $\varphi$ on $X \times$ $Y$, linear transformations $S_{\varphi}$ on $\mathcal{B}\left(H_{1}, H_{2}\right)$; these transformations are called Schur multipliers or, in the more general setting of spectral measures in the place of $\mu$ and $\nu$, double operator integrals. Namely, one first defines the map $S_{\varphi}$ on the space of all Hilbert-Schmidt operators by multiplying their integral kernels by $\varphi$; if $S_{\varphi}$ is bounded in the operator norm, one extends it

The second author was supported by EPSRC grant D050677/1. The third author was supported by the Swedish Research Council. 
to the space $\mathcal{K}\left(H_{1}, H_{2}\right)$ of all compact operators by continuity. The map $S_{\varphi}$ is defined on $\mathcal{B}\left(H_{1}, H_{2}\right)$ by taking the second dual of the constructed map on $\mathcal{K}\left(H_{1}, H_{2}\right)$.

The function $\varphi$ is thus called a Schur multiplier if the multiplication map $S_{\varphi}$, initially defined on the space of Hilbert-Schmidt operators, is bounded in the operator norm. Equivalently, $\varphi$ is a Schur multiplier if $\varphi(x, y) k(x, y)$ is the integral kernel of a nuclear operator provided $k(x, y)$ is such. The set of all Schur multipliers will be denoted by $\mathfrak{S}(X, Y)$.

Many years ago, Professor Solomyak informed the first author that at the early stages of the development of the theory of double operator integrals, there existed an idea to define $S_{\varphi}$ for a more general class of functions $\varphi$ (perhaps for all measurable ones) as the closure of a densely defined linear operator in the operator norm, or in the weak operator, topology. However, this approach was not pursued because no information on the closability of the multiplication maps $S_{\varphi}$ was obtained at that time.

The aim of this paper is to study the classes of functions $\varphi$ for which $S_{\varphi}$ is closable in the norm topology, or in the weak* topology, of $\mathcal{B}\left(H_{1}, H_{2}\right)$. By weak* topology we mean the topology $\sigma\left(\mathcal{B}\left(H_{1}, H_{2}\right), \mathcal{C}_{1}\left(H_{2}, H_{1}\right)\right)$ induced on $\mathcal{B}\left(H_{1}, H_{2}\right)$ by its duality with the space $\mathcal{C}_{1}\left(H_{2}, H_{1}\right)$ of nuclear operators from $H_{2}$ into $H_{1}$. We denote these classes of functions by $\mathfrak{S}_{\mathrm{cl}}(X, Y)$ and $\mathfrak{S}_{w^{*}}(X, Y)$, respectively.

We obtain a satisfactory characterisation of the class $\mathfrak{S}_{w^{*}}(X, Y)$. In order to describe it, let us denote by $\mathfrak{S}_{\text {loc }}(X, Y)$ the class of functions $\varphi$ with the following property: for each $\varepsilon>0$ there exist subsets $X_{\varepsilon} \subseteq X$ and $Y_{\varepsilon} \subseteq Y$ of measure not exceeding $\varepsilon$, such that the restriction of $\varphi$ to $\left(X \backslash X_{\varepsilon}\right) \times\left(Y \backslash Y_{\varepsilon}\right)$ is a Schur multiplier. We prove in Theorem 3.6 that the elements of $\mathfrak{S}_{\text {loc }}(X, Y)$ (which we call local Schur multipliers) can be characterised in terms similar to those of Peller's characterisation of Schur multipliers [26]. Namely, they are precisely the functions of the form $(a(x), b(y))$, where $a(\cdot)$ (resp. $b(\cdot))$ is a measurable function from $X$ (resp. $Y$ ) into a separable Hilbert space. Then we show in Theorem 4.4 that the $\mathrm{w}^{*}$-closable multipliers (that is, the elements of $\left.\mathfrak{S}_{w^{*}}(X, Y)\right)$ are precisely the functions of the form $t(x, y) / s(x, y)$ where $t$ and $s$ are local Schur multipliers and $s(x, y) \neq 0$ for marginally almost all $(x, y)$. In particular, $\mathfrak{S}_{w^{*}}(X, Y)$ is an algebra of (equivalent classes of) functions.

For any measurable function $\varphi$ on $X \times Y$, there exists a maximal (in a sense that we make precise in Section 4) countable family of rectangles on each of which $\varphi$ is $\mathrm{w}^{*}$-closable; the complement of their union is denoted by $\kappa_{\varphi}^{w^{*}}$. The "size" of $\kappa_{\varphi}^{w^{*}}$ can be considered as a measure of the extent to which $\varphi$ fails to be a $\mathrm{w}^{*}$-closable multiplier.

The information we obtain about $\mathfrak{S}_{\mathrm{cl}}(X, Y)$ is less precise. Roughly speaking, we show that, in order to verify whether $\varphi$ belongs to $\mathfrak{S}_{\mathrm{cl}}(X, Y)$, one needs to check whether the set $\kappa_{\varphi}^{w^{*}}$ supports a non-zero compact operator. More precisely: if $\kappa_{\varphi}^{w^{*}}$ does not support a non-zero compact operator then $\varphi \in$ $\mathfrak{S}_{\mathrm{cl}}(X, Y)$ while if there exists a non-zero compact operator in the smallest 
masa-bimodule with support $\kappa_{\varphi}^{w^{*}}$ then $\varphi \notin \mathfrak{S}_{\mathrm{cl}}(X, Y)$. The difference between the smallest masa-bimodule with support $\kappa_{\varphi}^{w^{*}}$ and the largest one (the set of all operators supported on $\kappa_{\varphi}^{w^{*}}$ ) is subtle; it is the subject of the theory of operator synthesis [1,28], an operator analogue of the theory of spectral synthesis $[15,20]$. We prove that $\mathfrak{S}_{\mathrm{cl}}(X, Y)$ is an algebra and present various examples of multipliers which are not norm-closable and of norm-closable multipliers which are not $\mathrm{w}^{*}$-closable.

The product of two measure spaces possesses natural "pseudo-topological structures", namely the $\omega$-topology and the $\tau$-topology, which are related to the problem of closability of multipliers. A set is called $\tau$-open (resp. $\omega$-open) if it is a countable union of measurable rectangles and a null set (resp. a set contained in $\left(X_{0} \times Y\right) \cup\left(X \times Y_{0}\right)$, where $X_{0}$ and $Y_{0}$ are null sets). Denoting by $C_{\tau}(X \times Y)$ (resp. $\left.C_{\omega}(X \times Y)\right)$ the space of all $\tau$-continuous (resp. $\omega$-continuous) complex valued functions on $X \times Y$, we prove that

$$
\mathfrak{S}_{\mathrm{cl}}(X, Y) \subseteq C_{\tau}(X \times Y) \text { and } \mathfrak{S}_{w^{*}}(X, Y) \subseteq C_{\omega}(X \times Y)
$$

(if one identifies functions equivalent with respect to the product measure). Both inclusions are shown to be strict.

We present examples which show that in the chain

$$
\mathfrak{S}(X, Y) \subseteq \mathfrak{S}_{\mathrm{loc}}(X, Y) \subseteq \mathfrak{S}_{w^{*}}(X, Y) \subseteq \mathfrak{S}_{\mathrm{cl}}(X, Y)
$$

the first and the third inclusions are strict. The question of whether the second inclusion is strict is left open.

The paper is organised as follows. In Section 2 we state some basic definitions and results about subsets of, and functions on, product measure spaces, bimodules over maximal abelian selfadjoint algebras, Schur multipliers and closable operators. In Section 3 we examine local Schur multipliers, while Sections 4 and 5 are devoted to the study of $\mathrm{w}^{*}$-closable and norm-closable multipliers, respectively.

In Sections 6 and 7 we study multipliers of special types. Given a complex function $f$ defined on a subinterval of the real line, one may consider its divided difference, in other words, the function $\check{f}$ on two variables given by $\check{f}(x, y)=(f(x)-f(y)) /(x-y)$. The corresponding class of multipliers plays an important role in Perturbation Theory and Spectral Theory (see, for example, [26] and the references therein). We show that such multipliers are always norm-closable; in Theorem 6.3 and Corollary 6.4 we formulate necessary and sufficient conditions for $\check{f}$ to be a local Schur multiplier.

Toeplitz multipliers are (Schur, local, norm-closable or $\mathrm{w}^{*}$-closable) multipliers $\varphi$ of the form $\varphi(x, y)=f(x-y)$, where $f$ is a complex function defined on a locally compact abelian group $G$ (equipped with Haar measure $\mu$ ). Theorem 7.8 asserts that a function $f(x-y)$ is a $\mathrm{w}^{*}$-closable multiplier if and only if it is a local Schur multiplier, and that this occurs precisely when $f$ is equivalent to a function which belongs locally to the Fourier algebra of $G$. The closability of Toeplitz multipliers is shown to be related to some questions about sets of multiplicity in harmonic analysis. We describe also those functions of the form $f(x-y)$ which are $((\mu \times \mu)$-equivalent to $) \omega$-continuous 
or $\tau$-continuous functions. En route, we obtain an example of a continuous (hence $\omega$-continuous) function on $G \times G$ which is not a norm-closable multiplier.

Acknowledgements. The authors are very grateful to M. Roginskaya and T. W. Körner for their friendly and helpful advice, and to the referee for suggestion to consider a $\mathcal{C}_{p}$-version of Problem 4 (see Remark 8.1(i)).

\section{Preliminary results}

\subsection{Pseudo-topologies on the product of measure spaces}

In what follows, we write $\mathfrak{B}(Z)=\mathfrak{B}(Z, \gamma)$ for the algebra, with respect to the pointwise product, of all measurable complex valued functions defined on a measure space $(Z, \gamma)$. Let $(X, \mu)$ and $(Y, \nu)$ be standard $\sigma$-finite measure spaces, which will be fixed throughout the paper. We will often write $\mathfrak{B}(X \times$ $Y)$ in the place of $\mathfrak{B}(X \times Y, \mu \times \nu)$.

A subset of $X \times Y$ is said to be a measurable rectangle (or simply a rectangle) if it is of the form $\alpha \times \beta$, where $\alpha \subseteq X$ and $\beta \subseteq Y$ are measurable subsets. A subset $E \subseteq X \times Y$ is called marginally null if $E \subseteq\left(X_{0} \times Y\right) \cup$ $\left(X \times Y_{0}\right)$, where $\mu\left(X_{0}\right)=\nu\left(Y_{0}\right)=0$. We call two subsets $E, F \subseteq X \times Y$ marginally equivalent (and write $E \simeq F$ ) if the symmetric difference of $E$ and $F$ is marginally null. We say that $E$ marginally contains $F$ (or $F$ is marginally contained in $E$ ) if $F \backslash E$ is marginally null; $E$ and $F$ are said to be marginally disjoint if $E \cap F$ is marginally null.

A subset $E$ of $X \times Y$ is called $\omega$-open if it is marginally equivalent to the union of a countable set of rectangles. The complements of $\omega$-open sets are called $\omega$-closed. It is clear that the class of all $\omega$-open (resp. $\omega$-closed) sets is closed under countable unions (resp. intersections) and finite intersections (resp. unions); in other words, the $\omega$-open sets form a pseudo-topology.

The following lemma shows that in some cases one can form a certain kind of a union of a given, possibly uncountable, family of $\omega$-open subsets.

Lemma 2.1. Let $\mathcal{E}$ be a family of $\omega$-open subsets of $X \times Y$. Let $\mathcal{E}_{\sigma}$ be the set of all countable unions of elements of $\mathcal{E}$. Then there exists a (unique up to marginal equivalence) set $E \in \mathcal{E}_{\sigma}$ which marginally contains every set in $\mathcal{E}$.

Proof. First assume that the measures $\mu$ and $\nu$ are finite. On the set $\Pi$ of all measurable rectangles we introduce a metric $\rho$, setting, for $R_{1}=X_{1} \times Y_{1}$, $R_{2}=X_{2} \times Y_{2}$ in $\Pi, \rho\left(R_{1}, R_{2}\right)=\mu\left(X_{1} \nabla X_{2}\right)+\nu\left(Y_{1} \nabla Y_{2}\right)$ (here $\nabla$ denotes symmetric difference). Then $\Pi$ is a separable metric space whence the set $\mathcal{F}$ of all rectangles that are contained in elements of $\mathcal{E}$ is also separable. Let $\left\{R_{n}: n \geq 1\right\}$ be a dense sequence in $\mathcal{F}$ and $E=\cup_{n=1}^{\infty} R_{n}$. Then it is clear that $E$ marginally contains all $R \in \mathcal{F}$ and therefore all $E \in \mathcal{E}$.

In the general case, let $X=\cup_{n=1}^{\infty} X_{n}$ and $Y=\cup_{m=1}^{\infty} Y_{m}$ with $\mu\left(X_{n}\right)<\infty$ and $\nu\left(Y_{m}\right)<\infty, n, m \in \mathbb{N}$. For each pair $n, m$, let $\mathcal{E}_{n, m}=\left\{E \cap\left(X_{n} \times Y_{m}\right)\right.$ : $E \in \mathcal{E}\}$, let $E_{n, m}$ be the $\omega$-union of $\mathcal{E}_{n, m}$, and set $E=\cup_{n, m=1}^{\infty} E_{n, m}$.

The uniqueness is obvious. 
The set whose existence is guaranteed by Lemma 2.1 will be called the $\omega$-union of $\mathcal{E}$.

We will say that two functions $\varphi, \psi \in \mathfrak{B}(X \times Y)$ are equivalent, and write $\varphi \sim \psi$, if the set $D=\{(x, y) \in X \times Y: \varphi(x, y) \neq \psi(x, y)\}$ is null with respect to the product measure. If $D$ is marginally null then we say that $\varphi$ and $\psi$ coincide marginally everywhere, or that they are marginally equivalent, and write $\varphi \simeq \psi$. By $L^{\infty}(X \times Y)$ we denote as usual the subalgebra of $\mathfrak{B}(X \times Y)$ of all (equivalence classes of) essentially bounded functions.

Let $E \subseteq X \times Y$ be an $\omega$-open set. A measurable function $\varphi: E \rightarrow \mathbb{C}$ is called $\omega$-continuous if the set $\varphi^{-1}(G)$ is $\omega$-open for every open subset $G \subseteq \mathbb{C}$. As in [10, Corollary 3.2] one can see that the set $C_{\omega}(E)$ of all $\omega$ continuous functions on $E$ is a subalgebra of $\mathfrak{B}(E)$. For an arbitrary set $\mathcal{M} \subseteq C_{\omega}(X \times Y) \subseteq \mathfrak{B}(X \times Y)$, we let its null set null $(\mathcal{M})$ be the complement of the $\omega$-union of the family $\mathcal{E}=\left\{h^{-1}(\mathbb{C} \backslash\{0\}): h \in \mathcal{M}\right\}$.

We will need the following simple result from [21] (see the remark after [21, Proposition 8.1]).

Lemma 2.2. Let $E \subseteq X \times Y$ be an $\omega$-open set and let $f: E \rightarrow \mathbb{C}$ be an $\omega$-continuous function. If $f \sim 0$ then $f \simeq 0$.

Thus if two $\omega$-continuous functions are equivalent then they are marginally equivalent, and if a function is equivalent to an $\omega$-continuous function then the latter is defined uniquely up to marginal equivalence.

We will also need another pseudo-topology on $X \times Y$. Two subsets $E_{1}$, $E_{2}$ of $X \times Y$ will be called $\mu \times \nu$-equivalent if their symmetric difference is a $\mu \times \nu$-null set. We will say that a subset $E \subseteq X \times Y$ is $\tau$-open if it is $\mu \times \nu$-equivalent to a countable union of rectangles. It is clear that the pseudo-topology $\tau$ is stronger than $\omega$.

\subsection{Bimodules}

If $H_{1}$ and $H_{2}$ are Hilbert spaces, we denote by $\mathcal{B}\left(H_{1}, H_{2}\right)$ the space of all bounded linear operators from $H_{1}$ into $H_{2}$, and by $\mathcal{K}\left(H_{1}, H_{2}\right)$ (resp. $\left.\mathcal{C}_{1}\left(H_{1}, H_{2}\right), \mathcal{C}_{2}\left(H_{1}, H_{2}\right)\right)$ the space of compact (resp. nuclear, Hilbert-Schmidt) operators in $\mathcal{B}\left(H_{1}, H_{2}\right)$. Let $\|T\|_{\text {op }}$ denote the operator norm of $T \in \mathcal{B}\left(H_{1}, H_{2}\right)$. As usual, we write $\mathcal{B}(H)=\mathcal{B}(H, H)$. The space $\mathcal{C}_{1}\left(H_{2}, H_{1}\right)\left(\operatorname{resp} . \mathcal{B}\left(H_{1}, H_{2}\right)\right)$ can be naturally identified with the Banach space dual of $\mathcal{K}\left(H_{1}, H_{2}\right)$ (resp. $\mathcal{C}_{1}\left(H_{2}, H_{1}\right)$ ), the duality being given by the map $(T, S) \rightarrow\langle T, S\rangle \stackrel{\text { def }}{=} \operatorname{tr}(T S)$. Here $\operatorname{tr} A$ denotes the trace of a nuclear operator $A$. For a subset $\mathcal{W} \subseteq$ $\mathcal{C}_{1}\left(H_{2}, H_{1}\right)$, let $\mathcal{W}^{\perp}=\left\{T \in \mathcal{B}\left(H_{1}, H_{2}\right):\langle T, S\rangle=0\right.$, for each $\left.S \in \mathcal{W}\right\}$.

For the rest of the paper we let $H_{1}=L^{2}(X, \mu)$ and $H_{2}=L^{2}(Y, \nu)$. The space $L^{2}(X \times Y)$ will be identified with $\mathcal{C}_{2}\left(H_{1}, H_{2}\right)$ via the map sending an element $k \in L^{2}(X \times Y)$ to the integral operator $I_{k}$ given by $I_{k} \xi(y)=$ $\int_{X} k(x, y) \xi(x) d \mu(x), \xi \in H_{1}, y \in Y$. In a similar fashion, $\mathcal{C}_{1}\left(H_{2}, H_{1}\right)$ will be identified with the space $\Gamma(X, Y)$ of all functions $F: X \times Y \rightarrow \mathbb{C}$ which admit 
a representation

$$
F(x, y)=\sum_{i=1}^{\infty} f_{i}(x) g_{i}(y),
$$

where $f_{i} \in L^{2}(X, \mu), g_{i} \in L^{2}(Y, \nu), i \in \mathbb{N}, \sum_{i=1}^{\infty}\left\|f_{i}\right\|_{2}^{2}<\infty$ and $\sum_{i=1}^{\infty}\left\|g_{i}\right\|_{2}^{2}<$ $\infty$. Equivalently, $\Gamma(X, Y)$ can be defined as the projective tensor product $L^{2}(X, \mu) \hat{\otimes} L^{2}(Y, \nu)$. It was shown in [10, Theorem 6.5] that $\Gamma(X, Y)$ consists of $\omega$-continuous functions. For brevity, we often identify a function $h \in \Gamma(X, Y)$ with the corresponding integral operator $I_{h} \in \mathcal{C}_{1}\left(H_{2}, H_{1}\right)$. It will be convenient to write $\Gamma(X \times Y)$ in the place of $\Gamma(X, Y)$ (this allows for example to write $\Gamma(\kappa)$, where $\kappa$ is a rectangle).

If $f \in L^{\infty}(X, \mu)$, let $M_{f} \in \mathcal{B}\left(H_{1}\right)$ denote the operator of multiplication by $f$. We will often identify the collection $\left\{M_{f}: f \in L^{\infty}(X, \mu)\right\}$ of all such operators with the function space $L^{\infty}(X, \mu)$. If $\alpha \subseteq X$ is measurable, we write $P(\alpha)=M_{\chi_{\alpha}}$ for the multiplication by the characteristic function of the set $\alpha$. Similar definitions and identifications are made for $L^{\infty}(Y, \nu)$. A subspace $\mathcal{W} \subseteq \mathcal{B}\left(H_{1}, H_{2}\right)$ will be called a bimodule if $M_{\psi} T M_{\varphi} \in \mathcal{W}$ for all $T \in \mathcal{W}$, $\varphi \in L^{\infty}(X, \mu)$ and $\psi \in L^{\infty}(Y, \nu)$. One defines bimodules in $\mathcal{B}\left(H_{2}, H_{1}\right)$ in a similar fashion.

We say that an $\omega$-closed subset $\kappa \subseteq X \times Y$ supports an operator $T \in \mathcal{B}\left(H_{1}, H_{2}\right)$ (or that $T$ is supported on $\kappa$ ) if $P(\beta) T P(\alpha)=0$ whenever $\alpha \times \beta$ is marginally disjoint from $\kappa$. For any subset $\mathcal{M} \subseteq \mathcal{B}\left(H_{1}, H_{2}\right)$, there exists a smallest (up to marginal equivalence) $\omega$-closed set supp $\mathcal{M}$ which supports every operator $T \in \mathcal{M}$ [10]. By [28], for any $\omega$-closed set $\kappa$ there exists a smallest (resp. largest) $\mathrm{w}^{*}$-closed bimodule $\mathfrak{M}_{\min }(\kappa)$ (resp. $\left.\mathfrak{M}_{\max }(\kappa)\right)$ with support $\kappa$ in the sense that if $\mathfrak{M} \subseteq \mathcal{B}\left(H_{1}, H_{2}\right)$ is a $w^{*}$-closed bimodule with supp $\mathfrak{M}=\kappa$ then $\mathfrak{M}_{\min }(\kappa) \subseteq \mathfrak{M} \subseteq \mathfrak{M}_{\max }(\kappa)$. If $\mathfrak{M}_{\min }(\kappa)=\mathfrak{M}_{\max }(\kappa)$, the set $\kappa$ is called synthetic. By $\left[28\right.$, Theorem 4.4], $\mathfrak{M}_{\min }(\kappa)=\left\{I_{h}: h \in \Gamma(X, Y), h \chi_{\kappa} \simeq 0\right\}^{\perp}$.

Lemma 2.3. Let $\mathcal{W} \subseteq \mathcal{C}_{1}\left(H_{2}, H_{1}\right)$ be a bimodule and $\left\{f_{n}\right\}_{n=1}^{\infty} \subseteq \Gamma(X, Y)$ be a sequence such that $\left\{I_{f_{n}}\right\}_{n=1}^{\infty}$ is dense in $\mathcal{W}$. Then $\operatorname{null}(\mathcal{W})=\operatorname{supp}\left(\mathcal{W}^{\perp}\right)=$ $\cap_{n=1}^{\infty} f_{n}^{-1}(0)$.

In particular, $\mathcal{W}$ is norm dense in $\mathcal{C}_{1}\left(H_{2}, H_{1}\right)$ if and only if there exists a sequence $\left\{h_{n}\right\}_{n=1}^{\infty} \subseteq \Gamma(X, Y)$ such that $I_{h_{n}} \in \mathcal{W}$ for every $n \in \mathbb{N}$ and the set $\cap_{n=1}^{\infty} h_{n}^{-1}(0)$ is marginally null.

Proof. We start by showing the second statement. Since the Hilbert spaces $H_{1}$ and $H_{2}$ are separable, the space $\mathcal{C}_{1}\left(H_{2}, H_{1}\right)$ is separable and hence there exists a sequence $\left\{K_{n}\right\}_{n=1}^{\infty}$ dense in $\mathcal{W}$. Suppose that $K_{n}=I_{h_{n}}$, where $h_{n} \in$ $\Gamma(X, Y)$ and let $E=\cap_{n} h_{n}^{-1}(0)$. If $E$ is not marginally null then, by [28, Corollary 4.1], $\mathfrak{M}_{\min }(E)$ contains a non-zero operator $T$. By [28, Theorem 4.4], $\left\langle T, K_{n}\right\rangle=0$ for all $n \in \mathbb{N}$, and hence $\mathcal{W}$ is not dense.

Conversely, suppose that $\mathcal{W}$ is not dense, let $h_{n} \in \Gamma, n \in \mathbb{N}$, be such that $I_{h_{n}} \in \mathcal{W}$ and set $E=\cap_{n=1}^{\infty} h_{n}^{-1}(0)$. The annihilator $\mathfrak{M}$ of $\mathcal{W}$ in $\mathcal{B}\left(H_{1}, H_{2}\right)$ is a non-zero ${ }^{*}$-closed bimodule. By [28], the support $F$ of $\mathfrak{M}$ is not marginally null, and if an operator $I_{h}$ belongs to its preannihilator then $h$ vanishes 
marginally almost everywhere on $F$. It follows that $F$ is marginally contained in $E$ and hence $E$ is not marginally null.

Now we prove the general statement. It was shown in [28] (see the proof of $\left[28\right.$, Theorem 2.1]) that $\operatorname{null}(\overline{\mathcal{W}})=\operatorname{supp}\left(\mathcal{W}^{\perp}\right)$ where $\overline{\mathcal{W}}$ is the norm closure of $\mathcal{W}$ in $\mathcal{C}_{1}\left(H_{2}, H_{1}\right)$. Hence, up to marginally null sets, $\operatorname{supp}\left(\mathcal{W}^{\perp}\right) \subseteq$ $\operatorname{null}(\mathcal{W}) \subseteq \cap_{n=1}^{\infty} f_{n}^{-1}(0)$ and it suffices to show that $\cap_{n=1}^{\infty} f_{n}^{-1}(0) \subseteq \operatorname{supp}\left(\mathcal{W}^{\perp}\right)$. Let $\kappa$ be a rectangle disjoint from $\operatorname{supp}\left(\mathcal{W}^{\perp}\right)$. By the definition of support, the restriction to $\kappa$ of the functions corresponding to operators in $\mathcal{W}$ form a set dense in $\Gamma(\kappa)$. By the first part of the proof, the intersection of the null sets of the restrictions of $f_{n}, n \in \mathbb{N}$, to $\kappa$ is marginally null. Hence $\kappa$ is marginally disjoint from $\cap_{n=1}^{\infty} f_{n}^{-1}(0)$. Since the complement of $\operatorname{supp}\left(\mathcal{W}^{\perp}\right)$ is $\omega$-closed, this implies the last remaining inclusion.

\subsection{Schur multipliers and Peller's Theorem}

For a function $\varphi \in \mathfrak{B}(X \times Y)$, set

$$
D(\varphi)=\left\{k \in L^{2}(X \times Y): \varphi k \in L^{2}(X \times Y)\right\} .
$$

We let $S_{\varphi}: D(\varphi) \rightarrow L^{2}(X \times Y)$ be the mapping given by $S_{\varphi} k=\varphi k$. We identify $S_{\varphi}$ with a (densely defined linear) map on $\mathcal{K}\left(H_{1}, H_{2}\right)$ acting by the rule $S_{\varphi}\left(I_{k}\right)=I_{\varphi k}$.

Note that $S_{\varphi}$ depends only on the equivalence class of $\varphi$. Taking this into account, we will sometimes say that a function $\varphi$ belongs to a certain class of functions, if it is equivalent to a function from this class. When we need to make the distinction, we will write $h \in^{\sigma} \mathcal{F}$, if $h$ is equivalent to a function from the class $\mathcal{F}$ with respect to the measure $\sigma$.

Recall that a function $\varphi \in L^{\infty}(X \times Y)$ is called a Schur multiplier if the map $S_{\varphi}$ is bounded in the operator norm, that is, if there exists a constant $C>0$ such that $\left\|S_{\varphi}\left(I_{k}\right)\right\|_{\text {op }} \leq C\left\|I_{k}\right\|_{\text {op }}$, for all $k \in L^{2}(X \times Y)$. If $\varphi$ is a Schur multiplier then the mapping $S_{\varphi}$ has a unique weak* continuous extension to $\mathcal{B}\left(H_{1}, H_{2}\right)$ which will still be denoted by $S_{\varphi}$.

Let $\mathfrak{S}(X, Y)($ or $\mathfrak{S}(X \times Y))$ be the set of all Schur multipliers; clearly, $\mathfrak{S}(X, Y)$ is a subalgebra of $\mathfrak{B}(X, Y)$. The following facts follow easily from the definition of a Schur multiplier:

Lemma 2.4. (i) If $\varphi \in \mathfrak{S}(X \times Y)$ then $\left.\varphi\right|_{\alpha \times \beta} \in \mathfrak{S}(\alpha \times \beta)$ for all measurable subsets $\alpha \subseteq X$ and $\beta \subseteq Y$.

(ii) If $X \times Y=\cup_{p=1}^{N} \kappa_{p}$, where all $\kappa_{p}$ are rectangles and $\left.\varphi\right|_{\kappa_{p}} \in \mathfrak{S}\left(\kappa_{p}\right)$ then $\varphi \in \mathfrak{S}(X, Y)$.

Schur multipliers were first introduced by Schur in the early 20th century in case of discrete measures $\mu$ and $\nu$. A characterisation of this particular class of Schur multipliers was obtained by Grothendieck in [16]. The following generalisation for the class defined above is due to Peller [26].

Theorem 2.5. Let $\varphi \in L^{\infty}(X \times Y)$. The following conditions are equivalent: (i) $\varphi$ is a Schur multiplier; 

that

(ii) there exist measurable functions $a: X \rightarrow l^{2}$ and $b: Y \rightarrow l^{2}$ such $\varphi(x, y)=(a(x), b(y))_{l^{2}}$, a.e. on $X \times Y$ and $\sup _{x \in X}\|a(x)\|_{2} \sup _{y \in Y}\|b(y)\|_{2}<\infty$.

(iii) $\varphi(x, y) k(x, y) \in^{\mu \times \nu} \Gamma(X, Y)$ whenever $k(x, y) \in \Gamma(X, Y)$.

It follows from Peller's Theorem (and can easily be seen directly) that if the measures $\mu$ and $\nu$ are finite then $\mathfrak{S}(X, Y) \subseteq \Gamma(X, Y)$.

Using modern terminology, one can say that Theorem 2.5 identifies the algebra $\mathfrak{S}(X, Y)$ with the weak* Haagerup tensor product $L^{\infty}(X, \mu) \otimes_{\mathrm{w}^{*} \mathrm{~h}}$ $L^{\infty}(Y, \nu)$ (see [6] where this tensor product was introduced).

\subsection{General facts on closable operators}

Let $\mathcal{X}$ be a Banach space. We denote by $\mathcal{X}^{*}$ the dual of $\mathcal{X}$. If $\mathcal{S} \subseteq \mathcal{X}$ (resp. $\left.\mathcal{T} \subseteq \mathcal{X}^{*}\right)$, we write $\mathcal{S}^{\perp} \subseteq \mathcal{X}^{*}$ (resp. $\mathcal{T}_{\perp} \subseteq \mathcal{X}$ ) for the annihilator (resp. preannihilator) of $\mathcal{S}$ (resp. $\mathcal{T}$ ).

Let $\mathcal{Y}$ be another Banach space. By an operator from $\mathcal{X}$ into $\mathcal{Y}$ we mean a linear transformation $T: D(T) \rightarrow \mathcal{Y}$, where $D(T)$ is a (not necessarily closed) linear subspace of $\mathcal{X}$ called the domain of $T$. The operator $T$ is called densely defined if $D(T)$ is norm dense in $\mathcal{X}$. We let

$$
\operatorname{Gr} T=\{(x, T x): x \in D(T)\} \subseteq \mathcal{X} \oplus \mathcal{Y}
$$

be the graph of $T$. For a subset $\mathcal{S} \subseteq \mathcal{Y} \oplus \mathcal{X}$ we set $\mathcal{S}^{\prime}=\{(x, y):(y, x) \in \mathcal{S}\}$ and let $\mathrm{Gr}^{\prime} T=(\mathrm{Gr} T)^{\prime}$.

We recall the definition of the adjoint $T^{*}$ of an operator $T: D(T) \rightarrow \mathcal{Y}$. The domain of $T^{*}$ is the subspace

$$
D\left(T^{*}\right)=\left\{g \in \mathcal{Y}^{*}: \exists f \in \mathcal{X}^{*} \text { such that } g(T x)=f(x), \forall x \in D(T)\right\} .
$$

For $g \in D\left(T^{*}\right)$, one lets $T^{*} g$ equal to $f$ where $f \in \mathcal{X}^{*}$ is the functional appearing in the definition of $D\left(T^{*}\right)$. Note that $g \in D\left(T^{*}\right)$ if and only if the linear map $x \rightarrow g(T x)$ from $D(T)$ into $\mathbb{C}$ is continuous. By the definition of the operator $T^{*}$, we have that $\mathrm{Gr}^{\prime}\left(-T^{*}\right)=(\operatorname{Gr} T)^{\perp}$.

Recall that an operator $T$ is called closable if the norm closed hull $\overline{\operatorname{Gr} T}$ of its graph is the graph of an operator. Clearly, $T$ is closable if and only if the conditions $\left(x_{n}\right)_{n=1}^{\infty} \subseteq \mathcal{X}, y \in \mathcal{Y},\left\|x_{n}\right\| \rightarrow 0$ and $\left\|T x_{n}-y\right\| \rightarrow 0$ imply that $y=0$. We call $T w^{*}$-closable if the $\mathrm{w}^{*}$-closed hull $\overline{\mathrm{Gr} T} \bar{w}^{w^{*}} \subseteq \mathcal{X}^{* *} \oplus \mathcal{Y}^{* *}$ of its graph is the graph of an operator from $\mathcal{X}^{* *}$ into $\mathcal{Y}^{* *}$. Here, we identify $\mathcal{X}$ and $\mathcal{Y}$ with their canonical images in their second duals. We have that $T$ is $\mathrm{w}^{*}$-closable if and only if the conditions $\left(x_{\alpha}\right)_{\alpha} \subseteq \mathcal{X}, G \in \mathcal{Y}^{* *}, \mathrm{w}$ - $\lim _{\alpha} x_{\alpha}=0$ and $\mathrm{w}^{*}-\lim T x_{\alpha}=G$ imply that $G=0$. The weak* limit is taken with respect to the weak* topology of $\mathcal{Y}^{* *}$.

In the following proposition the equivalence (iii) $\Leftrightarrow$ (iv) is well-known (see, for example, [19, Chapter III, Section 5]); the other implications can be proved in a similar way. 
Proposition 2.6. Let $T: D(T) \rightarrow \mathcal{Y}$ be a densely defined linear operator and set $\mathcal{D}=D\left(T^{*}\right)$. Consider the following conditions:

(i) $T$ is $w^{*}$-closable;

(ii) $\overline{\mathcal{D}}^{\|\cdot\|}=\mathcal{Y}^{*}$;

(iii) $\overline{\mathcal{D}}^{w^{*}}=\mathcal{Y}^{*}$;

(iv) $T$ is closable.

Then $(i) \Longleftrightarrow(i i) \Longrightarrow(i i i) \Longleftrightarrow(i v)$.

\section{Local Schur multipliers}

We start this section by introducing a class of functions that will play a central role in the paper. For brevity, let us say that a countable family of rectangles covers $X \times Y$, or that it is a covering family, if its union is marginally equivalent to $X \times Y$.

Definition 3.1. A function $\varphi \in \mathfrak{B}(X \times Y)$ will be called a local Schur multiplier if there exists a covering family $\left\{\kappa_{m}\right\}_{m=1}^{\infty}$ of rectangles in $X \times Y$ such that $\left.\varphi\right|_{\kappa_{m}} \in \mathfrak{S}\left(\kappa_{m}\right)$, for each $m \in \mathbb{N}$.

The set of all local Schur multipliers on $X \times Y$ will be denoted by $\mathfrak{S}_{\text {loc }}(X, Y)$.

Proposition 3.2. The set $\mathfrak{S}_{\text {loc }}(X, Y)$ is a subalgebra of the algebra $C_{\omega}(X, Y)$ of all $\omega$-continuous functions.

Proof. Let $\varphi \in \mathfrak{S}_{\text {loc }}(X, Y)$. By the $\sigma$-finiteness of the measure spaces $(X, \mu)$ and $(Y, \nu)$, there exists a covering family $\left\{\kappa_{m}\right\}_{m=1}^{\infty}$ such that $\left.\varphi\right|_{\kappa_{m}} \in \mathfrak{S}\left(\kappa_{m}\right)$, and $(\mu \times \nu)\left(\kappa_{m}\right)<\infty$ for each $m \in \mathbb{N}$. It follows that $\left.\varphi\right|_{\kappa_{m}} \in \Gamma\left(\kappa_{m}\right)$. By [10, Theorem 6.5], $\left.\varphi\right|_{\kappa_{m}}$ is $\omega$-continuous on $\kappa_{m}, m \in \mathbb{N}$. Now for an open subset $G \subseteq \mathbb{C}$, we have that $\varphi^{-1}(G)=\cup_{m=1}^{\infty}\left(\kappa_{m} \cap \varphi^{-1}(G)\right)$ is $\omega$-open, and hence $\varphi$ is $\omega$-continuous.

It is easy to see that for two functions $\varphi, \psi$ in $\mathfrak{S}_{\text {loc }}(X, Y)$, one can find a common covering family $\left\{\kappa_{m}\right\}_{m=1}^{\infty}$ of rectangles on which both $\varphi$ and $\psi$ are Schur multipliers. Since $\mathfrak{S}\left(\kappa_{m}\right)$ is an algebra, $\varphi+\psi$ and $\varphi \psi$ belong to $\mathfrak{S}_{\text {loc }}(X, Y)$.

Let $\mathcal{V}(X, Y)$ be the space of all functions $\varphi \in \mathfrak{B}(X \times Y)$ for which there exist families $\left\{a_{i}\right\}_{i=1}^{\infty} \subseteq \mathfrak{B}(X)$ and $\left\{b_{i}\right\}_{i=1}^{\infty} \subseteq \mathfrak{B}(Y)$ with the properties

$$
\sum_{i=1}^{\infty}\left|a_{i}(x)\right|^{2}<\infty, \quad \sum_{i=1}^{\infty}\left|b_{i}(y)\right|^{2}<\infty
$$

for almost all $x \in X$ and $y \in Y$, and

$$
\varphi(x, y)=\sum_{i=1}^{\infty} a_{i}(x) b_{i}(y), \text { almost everywhere on } X \times Y \text {. }
$$

Using a coordinate free language we may say that $\varphi \in \mathcal{V}(X, Y)$ if and only if there exists a separable Hilbert space $H$ and measurable functions $a: X \rightarrow H$ and $b: Y \rightarrow H$ such that $\varphi(x, y)=(a(x), b(y))$ for almost all $(x, y) \in X \times Y$. 
We note that $\Gamma(X, Y) \subseteq \mathcal{V}(X, Y)$ and $\mathfrak{S}(X, Y) \subseteq \mathcal{V}(X, Y)$. Indeed, these function spaces correspond to the cases where the functions $\|a(\cdot)\|,\|b(\cdot)\|$ are, respectively, square integrable and essentially bounded.

Lemma 3.3. If $\varphi \in \mathcal{V}(X, Y)$ then there exist families $\left\{X_{i}\right\}_{i=1}^{\infty}$ and $\left\{Y_{j}\right\}_{j=1}^{\infty}$ of pairwise disjoint subsets of $X$ and $Y$, respectively, such that $\left.\varphi\right|_{X_{i} \times Y_{j}} \in$ $\mathfrak{S}\left(X_{i}, Y_{j}\right)$, for all $i, j \in \mathbb{N}$. We may moreover assume that $\mu\left(X_{i}\right)<\infty$ and $\nu\left(Y_{j}\right)<\infty$, for all $i, j \in \mathbb{N}$.

Proof. Let $a: X \rightarrow \ell^{2}$ and $b: Y \rightarrow \ell^{2}$ be measurable functions such that $\varphi(x, y)=(a(x), b(y))$, for almost all $(x, y)$. For $i, j \in \mathbb{N}$, set $X_{i}=\{x \in X$ : $\left.i-1 \leq\|a(x)\|_{2}<i\right\}$ and $Y_{j}=\left\{y \in Y: j-1 \leq\|b(y)\|_{2}<j\right\}$. Then $\left.\varphi\right|_{X_{i} \times Y_{j}} \in \mathfrak{S}\left(X_{i}, Y_{j}\right)$ by Theorem 2.5. Partitioning $X_{i}$ and $Y_{j}$ into subsets of finite measure, we obtain the required decompositions.

Lemma 3.3 shows, in particular, that $\mathcal{V}(X, Y) \subseteq \mathfrak{S}_{\text {loc }}(X, Y)$. Our aim in this section is to show that, in fact, $\mathcal{V}(X, Y)=\mathfrak{S}_{\text {loc }}(X, Y)$.

Lemma 3.4. Let $\left\{\kappa_{m}\right\}_{m=1}^{\infty}$ be a covering sequence of $\omega$-open sets. Then there exist families $\left\{X_{i}\right\}_{i=1}^{\infty}$ and $\left\{Y_{j}\right\}_{j=1}^{\infty}$ of pairwise disjoint measurable subsets of $X$ and $Y$, respectively, such that

(i) $\cup_{i=1}^{\infty} X_{i}$ and $\cup_{j=1}^{\infty} Y_{j}$ have full measure, and

(ii) each rectangle $X_{i} \times Y_{j}$ is contained in a finite union of sets from $\left\{\kappa_{m}\right\}_{m=1}^{\infty}$.

Proof. Let us say that a subset $E \subseteq X \times Y$ is mild if it is contained in a finite union of sets from the family $\left\{\kappa_{m}\right\}_{m=1}^{\infty}$. It suffices to show that there are increasing sequences $\left\{A_{n}\right\}_{n=1}^{\infty}$ and $\left\{B_{n}\right\}_{n=1}^{\infty}$ of measurable subsets of $X$ and $Y$, respectively, such that $\cup_{n=1}^{\infty} A_{n}$ and $\cup_{n=1}^{\infty} B_{n}$ have full measure and all rectangles of the form $A_{n} \times B_{n}$ are mild. Indeed, the statement would then follow by setting $X_{i}=A_{i} \backslash A_{i-1}, Y_{j}=B_{j} \backslash B_{j-1}$.

Since the measure spaces $(X, \mu),(Y, \nu)$ are standard we may assume that $X$ and $Y$ are equipped with $\sigma$-compact topologies with respect to which $\mu$ and $\nu$ are regular Borel measures. By considering increasing sequences $\left\{U_{n}\right\}_{n=1}^{\infty}$ and $\left\{V_{n}\right\}_{n=1}^{\infty}$ of compact subsets of $X$ and $Y$, respectively, we reduce the problem to the case where $X$ and $Y$ are compact and $\mu$ and $\nu$ are finite.

We may clearly assume that each $\kappa_{m}$ is a rectangle. By [10, Lemma 3.4], for any $\epsilon>0$ there exists $X_{\epsilon} \subseteq X, Y_{\epsilon} \subseteq Y$ such that $\mu\left(X \backslash X_{\epsilon}\right)<\epsilon$, $\nu\left(Y \backslash Y_{\epsilon}\right)<\epsilon$ and $X_{\epsilon} \times Y_{\epsilon}$ is contained in a finite union of rectangles $\kappa_{m}$. Let $\epsilon_{n}=2^{-n}, L_{n}=\cap_{k=n}^{\infty} X_{\epsilon_{k}}$ and $M_{n}=\cap_{k=n}^{\infty} Y_{\epsilon_{k}}$. Then each $L_{n} \times M_{n}$ is mild since it is contained in $X_{\epsilon_{n}} \times Y_{\epsilon_{n}}$. Furthermore, $L_{n} \subseteq L_{n+1}, M_{n} \subseteq M_{n+1}$,

$\mu\left(X \backslash L_{n}\right) \leq \sum_{k=n}^{\infty} \mu\left(X \backslash X_{\epsilon_{k}}\right)<\epsilon 2^{2-n}$ and $\nu\left(Y \backslash M_{n}\right) \leq \sum_{k=n}^{\infty} \mu\left(Y \backslash Y_{\epsilon_{k}}\right)<\epsilon 2^{2-n}$.

Thus, $\mu\left(X \backslash\left(\cup_{n=1}^{\infty} L_{n}\right)\right)=0$ and $\nu\left(Y \backslash\left(\cup_{n=1}^{\infty} M_{n}\right)\right)=0$ and the proof is complete.

The following result may be viewed as an analogue of Lemma 2.4 for local multipliers. 
Lemma 3.5. Let $\left\{X_{i}\right\}_{i=1}^{\infty}$ and $\left\{Y_{j}\right\}_{j=1}^{\infty}$ be families of pairwise disjoint subsets of $X$ and $Y$, respectively, such that $X=\cup_{i=1}^{\infty} X_{i}$ and $Y=\cup_{j=1}^{\infty} Y_{j}$. Assume that $\varphi \in \mathfrak{B}(X \times Y)$ is such that $\left.\varphi\right|_{X_{i} \times Y_{j}} \in \mathfrak{S}\left(X_{i}, Y_{j}\right)$ for all $i, j \in \mathbb{N}$. Then $\varphi \in \mathcal{V}(X, Y)$.

Proof. Let $\varphi_{i, j}(x, y)=\left.\varphi\right|_{X_{i} \times Y_{j}}$. By our assumption, $\varphi_{i, j} \in \mathfrak{S}\left(X_{i}, Y_{j}\right)$ and hence, by Theorem 2.5, there exist measurable functions $a_{i, j}: X \rightarrow \ell^{2}$ and $b_{i, j}: Y \rightarrow \ell^{2}$ such that $\varphi(x, y)=\left(a_{i, j}(x), b_{i, j}(y)\right)$ for almost all $(x, y) \in$ $X_{i} \times Y_{j}$ and

$$
\alpha_{i, j} \stackrel{\text { def }}{=} \sup _{x \in X_{i}}\left\|a_{i j}(x)\right\|_{2} \sup _{y \in Y_{j}}\left\|b_{i j}(y)\right\|_{2}<\infty .
$$

We may clearly assume that $\sup _{x \in X_{i}}\left\|a_{i j}(x)\right\|_{2}=\sup _{y \in Y_{j}}\left\|b_{i j}(y)\right\|_{2}$. Let $H=$ $\oplus_{i, j} H_{i, j}$, where $H_{i, j}=\ell^{2}$ for all $i, j \in \mathbb{N}$. Considering $a_{i, j}(x)$ as a vector in $H_{i, j}$, we define a function $a: X \rightarrow H$ in the following way: if $x \in X_{k}$ then set $a(x)=\oplus_{i, j} \xi_{i, j}(x)$, where $\xi_{k, j}(x)=a_{k, j}(x) / j \sqrt{\alpha_{k, j}}$ and $\xi_{i, j}(x)=0$ for $i \neq k$. Similarly, we define $b: Y \rightarrow H$ by letting, for $y \in Y_{l}, b(y)=\oplus_{i, j} \eta_{i, j}(y)$, where $\eta_{i, l}(y)=b_{i, l}(y) / i \sqrt{\alpha_{i, l}}$ and $\eta_{i, j}(y)=0$ if $j \neq l$. Then for each $i$ and $x \in X_{i}$ we have

$$
\|a(x)\|_{H}^{2}=\sum_{j=1}^{\infty} \frac{\left\|a_{i, j}(x)\right\|_{2}^{2}}{j^{2} \alpha_{i, j}} \leq C,
$$

where $C=\sum_{j=1}^{\infty} \frac{1}{j^{2}}$. Similarly, we see that $\|b(y)\|_{H}^{2} \leq C$. Moreover, for $x \in X_{i}$ and $y \in Y_{j}$, we have that

$$
(a(x), b(y))_{H}=\frac{\left(a_{i, j}(x), b_{i, j}(y)\right)_{H_{i, j}}}{i j \alpha_{i, j}}
$$

and therefore

$$
\varphi(x, y) \chi_{X_{i} \times Y_{j}}=i j \alpha_{i, j}(a(x), b(y))_{H} \text {, for almost all }(x, y) \in X_{i} \times Y_{j} .
$$

The next step is to see that there exist families $\left\{p_{i}\right\}_{i=1}^{\infty}$ and $\left\{q_{j}\right\}_{j=1}^{\infty}$ of vectors in $\ell^{2}$ such that $\alpha_{i, j}=\left(p_{i}, q_{j}\right)_{\ell^{2}}, i, j \in \mathbb{N}$. We note first that $\left|\alpha_{i, j}\right| \leq$ $c_{i} c_{j}$, where $c_{i}=\max \left\{1,\left|\alpha_{k, l}\right|: k, l \leq i\right\}$ and $\frac{\alpha_{i, j}}{j c_{i} c_{j}}=\left(s_{i}, r_{j}\right)_{\ell^{2}}$, where $r_{j}=e_{j}$, $s_{i}=\sum_{j} \frac{\alpha_{i, j}}{j c_{i} c_{j}} e_{j}$ and $\left\{e_{j}\right\}_{j=1}^{\infty}$ is the standard basis of $\ell^{2}$. Observe that since $\sum_{j} \frac{\left|\alpha_{i, j}\right|^{2}}{j^{2} c_{i}^{2} c_{j}^{2}} \leq \sum_{j} \frac{1}{j^{2}}<\infty$, we have that $s_{i} \in \ell^{2}$. Setting $p_{i}=c_{i} s_{i}$ and $q_{j}=j c_{j} r_{j}$, we obtain $\alpha_{i, j}=\left(p_{i}, q_{j}\right)_{\ell_{2}}$. Now let $p(x)=i p_{i}$ if $x \in X_{i}$ and $q(y)=j q_{j}$ if $y \in Y_{j}$. Then

$$
\varphi(x, y)=(p(x), q(y))_{\ell^{2}}(a(x), b(y))_{H}=(p(x) \otimes a(x), q(y) \otimes b(y))_{\ell^{2} \otimes H}
$$

for almost all $(x, y) \in X \times Y$.

The following theorem is the main result of the present section. 
Theorem 3.6. Let $\varphi \in \mathfrak{B}(X \times Y)$. The following are equivalent:

(i) $\varphi$ is a local Schur multiplier;

(ii) $\varphi \in \mathcal{V}(X, Y)$.

Proof. (i) $\Rightarrow$ (ii) Let $\left\{\kappa_{m}\right\}_{m=1}^{\infty}$ be a covering family of rectangles from the definition of a local Schur multiplier. By Lemma 3.4, there exist families $\left\{X_{i}\right\}_{i=1}^{\infty}$ and $\left\{Y_{j}\right\}_{j=1}^{\infty}$ of pairwise disjoint measurable sets of $X$ and $Y$, respectively, whose unions have full measure and each rectangle $X_{i} \times Y_{j}$ is contained in a finite union of sets of the form $\kappa_{m}$.

Since $\left.\varphi\right|_{\kappa_{m}} \in \mathfrak{S}\left(\kappa_{m}\right)$ for all $m \in \mathbb{N}$, it follows from Lemma 2.4 that $\left.\varphi\right|_{X_{i} \times Y_{j}} \in \mathfrak{S}\left(X_{i} \times Y_{j}\right)$. An application of Lemma 3.5 implies (ii).

(ii) $\Rightarrow$ (i) follows from Lemma 3.3 .

Let $\mathcal{E}$ be the class of all rectangles $\alpha \times \beta$ such that $\left.\varphi\right|_{\alpha \times \beta}$ is a Schur multiplier. Let $\kappa_{\varphi}$ be the complement of the $\omega$-union of $\mathcal{E}$. Then $\kappa_{\varphi}$ is the smallest $\omega$-closed set with the property that $\varphi$ is a local Schur multiplier on each rectangle disjoint from it. We call $\kappa_{\varphi}$ the set of LM-singularity of $\varphi$ (LM is for "local multiplier"). It may be considered as a measure of how far $\varphi$ is from being a Schur multiplier. In particular, we say that $\varphi$ is extremely non-Schur multiplier if $\kappa_{\varphi}=X \times Y$. In Section 7 we will give an example of an $\omega$-continuous function which is extremely non-Schur multiplier.

\section{4. $\mathrm{w}^{*}$-closable multipliers}

We now introduce two classes of functions which, along with local Schur multipliers introduced in the previous section, are the main objects of study in the paper. We recall that $(X, \mu)$ and $(Y, \nu)$ are fixed standard measure spaces, $H_{1}=L^{2}(X, \mu)$ and $H_{2}=L^{2}(Y, \nu)$.

Definition 4.1. A function $\varphi \in \mathfrak{B}(X \times Y)$ is called a $w^{*}$-closable (resp. closable) multiplier if the map $S_{\varphi}$ is w $^{*}$-closable (resp. closable), when viewed as a densely defined linear operator on $\mathcal{K}\left(H_{1}, H_{2}\right)$.

For the sake of brevity, we will sometimes call a function $\mathrm{w}^{*}$-closable (resp. closable) if it is a $\mathrm{w}^{*}$-closable (resp. closable) multiplier. We recall that we denote by $\mathfrak{S}_{w^{*}}(X, Y)$ (resp. $\mathfrak{S}_{\mathrm{cl}}(X, Y)$ ) the set of all $\mathrm{w}^{*}$-closable (resp. closable) multipliers.

The operator $S_{\varphi}^{*}$ acting on $\mathcal{C}_{1}\left(H_{2}, H_{1}\right)$ can be easily described. Recall that the map $k \rightarrow I_{k}$ establishes an identification of $\Gamma(X, Y)$ with $\mathcal{C}_{1}\left(H_{2}, H_{1}\right)$ and that for $f \in \mathfrak{B}(X \times Y)$, we write $f \in^{\mu \times \nu} \Gamma(X, Y)$ if $f$ is $\mu \times \nu$-equivalent to a function in $\Gamma(X, Y)$.

Lemma 4.2. (i) We have that

$$
D\left(S_{\varphi}^{*}\right)=\left\{I_{h}: h \in \Gamma(X, Y) \text { and } \varphi h \in^{\mu \times \nu} \Gamma(X, Y)\right\} .
$$

In particular, $D\left(S_{\varphi}^{*}\right)$ is a bimodule.

(ii) For every $I_{h} \in D\left(S_{\varphi}^{*}\right)$, we have $S_{\varphi}^{*}\left(I_{h}\right)=I_{\varphi h}$. 
Proof. (i) For every $k \in L^{2}(X \times Y)$ and $h \in \Gamma(X, Y)$, we have $\left\langle I_{k}, I_{h}\right\rangle=$ $\int k h d(\mu \times \nu)$. It follows that a function $h \in \mathfrak{B}(X \times Y)$ is equivalent to a function in $\Gamma(X, Y)$ if and only if there exists $C>0$ such that

$$
\left|\int k h d(\mu \times \nu)\right| \leq C\left\|I_{k}\right\|_{\mathrm{op}}, \text { for all } k \in L^{2}(X \times Y) \text {. }
$$

We now have

$$
\begin{aligned}
I_{h} \in D\left(S_{\varphi}^{*}\right) & \Leftrightarrow\left|\left\langle S_{\varphi}\left(I_{k}\right), I_{h}\right\rangle\right| \leq C\left\|I_{k}\right\|_{\text {op }} \text { for all } k \in D\left(S_{\varphi}\right) \\
& \Leftrightarrow\left|\int \varphi k h d(\mu \times \nu)\right| \leq C\left\|I_{k}\right\|_{\text {op }} \text { for all } k \in D\left(S_{\varphi}\right) \\
& \Leftrightarrow \varphi h \in^{\mu \times \nu} \Gamma(X, Y),
\end{aligned}
$$

since $D\left(S_{\varphi}\right)$ is dense in $L^{2}(X \times Y)$.

(ii) is immediate from (i).

Lemma 4.3. Let $\varphi \in \mathfrak{B}(X \times Y)$. The following are equivalent:

(i) $\varphi$ is a $w^{*}$-closable multiplier;

(ii) there exists a covering family $\left\{\kappa_{m}\right\}_{m \in \mathbb{N}}$ of rectangles and functions $s_{m}, t_{m} \in \Gamma(X, Y)$ such that $s_{m}(x, y) \neq 0$ m.a.e. on $\kappa_{m}$ and

$$
\varphi(x, y)=\frac{t_{m}(x, y)}{s_{m}(x, y)}, \quad \text { a.e. on } \kappa_{m}, m \in \mathbb{N} .
$$

Proof. (i) $\Rightarrow$ (ii) If $\varphi$ is a $\mathrm{w}^{*}$-closable multiplier then, by Proposition 2.6, the subspace $\mathcal{U}=D\left(S_{\varphi}^{*}\right)$ is norm dense in $\mathcal{C}_{1}\left(H_{2}, H_{1}\right)$. By Lemma 2.3 , there is a sequence $\left\{h_{n}\right\}_{n=1}^{\infty} \subseteq \Gamma(X, Y)$ with $\left\{I_{h_{n}}\right\}_{n=1}^{\infty} \subseteq \mathcal{U}$ such that $\cap_{n=1}^{\infty} h_{n}^{-1}(0) \simeq \emptyset$. Hence, $\cup_{n=1}^{\infty} h_{n}^{-1}(\mathbb{C} \backslash\{0\}) \simeq X \times Y$. Since all $h_{n}$ are $\omega$-continuous, the sets $h_{n}^{-1}(\mathbb{C} \backslash\{0\})$ are $\omega$-open whence we may assume that they are countable unions of rectangles. We conclude that $X \times Y$ is marginally equivalent to a countable union of rectangles $\kappa_{m}, m \in \mathbb{N}$, such that for every $m \in \mathbb{N}$ there exists a function $s_{m} \in \mathcal{U}$ with $s_{m}(x, y) \neq 0$ on $\kappa_{m}$.

By Lemma 4.2, there exists a function $t_{m} \in \Gamma\left(\kappa_{m}\right)$ such that $t_{m} \sim \varphi s_{m}$. Hence $\varphi(x, y)=\frac{t_{m}(x, y)}{s_{m}(x, y)}$ almost everywhere on $\kappa_{m}$.

(ii) $\Rightarrow$ (i) Since $\varphi s_{m} \sim t_{m}$ and $t_{m} \in \Gamma(X, Y)$, Lemma 4.2 implies that $I_{s_{m}} \in D\left(S_{\varphi}^{*}\right)$. Since $\cap_{m=1}^{\infty} s_{m}^{-1}(0) \simeq \emptyset$, the space $D\left(S_{\varphi}^{*}\right)$ is norm dense in $\mathcal{C}_{1}\left(H_{2}, H_{1}\right)$ by Lemma 2.3. By Proposition 2.6, $S_{\varphi}$ is ${ }^{*}{ }^{*}$-closable.

The following characterisation of $\mathrm{w}^{*}$-closable multipliers is the main result of this section.

Theorem 4.4. A function $\varphi \in \mathfrak{B}(X \times Y)$ is a $w^{*}$-closable multiplier if and only if there exist functions $t, s \in \mathcal{V}(X, Y)$ such that $s(x, y) \neq 0$ marginally almost everywhere on $X \times Y$ and $\varphi(x, y)=\frac{t(x, y)}{s(x, y)}$, almost everywhere on $X \times Y$. 
Proof. Let $\varphi \in \mathfrak{B}(X \times Y)$ be a ${ }^{*}$-closable multiplier. By Lemma 4.3, there exists a covering family $\left\{\kappa_{m}\right\}_{m \in \mathbb{N}}$ of rectangles such that

$$
\varphi(x, y)=\frac{t_{m}(x, y)}{s_{m}(x, y)}, \quad \text { a.e. on } \kappa_{m},
$$

for some $s_{m}, t_{m} \in \Gamma(X, Y)$ with $s_{n}(x, y) \neq 0$ m.a.e. on $\kappa_{m}$.

Using Lemma 3.3 and the inclusion $\Gamma(X, Y) \subseteq \mathcal{V}(X, Y)$ we may, if necessary, partition the sets $\kappa_{m}$ into smaller rectangles and assume that the functions $t_{m}$ and $s_{m}$ belong to $\mathfrak{S}\left(\kappa_{m}\right)$.

By Lemma 3.4, there exist families $\left\{X_{k}\right\}_{k=1}^{\infty}$ and $\left\{Y_{l}\right\}_{l=1}^{\infty}$ of pairwise disjoint measurable subsets of $X$ and $Y$, respectively, such that $X=\cup_{k=1}^{\infty} X_{k}$, $Y=\cup_{l=1}^{\infty} Y_{l}$ and each $X_{k} \times Y_{l}$ is contained in a finite union of rectangles of the form $\kappa_{m}$. We show that on each rectangle $X_{k} \times Y_{l}$ the function $\varphi$ can be written in the form $\varphi(x, y)=\frac{t_{k, l}(x, y)}{s_{k, l}(x, y)}$ where $t_{k, l}, s_{k, l} \in \Gamma\left(X_{k}, Y_{l}\right)$ and $s_{k, l}(x, y) \neq 0$ marginally almost everywhere on $X_{k} \times Y_{l}$.

Indeed, $X_{k} \times Y_{l}$ is the union of a finite number of pairwise disjoint rectangles $Z \times W$ each of which is the intersections of some rectangles of the form $\kappa_{m}$ and $X_{k} \times Y_{l}$. Fix $(x, y) \in Z \times W$. On $Z \times W$ the function $\varphi$ can be written in the form $\frac{t_{0}}{s_{0}}$, where $t_{0}, s_{0} \in \Gamma(X, Y)$. We set $t_{k, l}(x, y)=t_{0}(x, y)$ and $s_{k, l}(x, y)=s_{0}(x, y)$.

Now let us define functions $s$ and $t$ on $X \times Y$ by setting $t(x, y)=t_{k, l}(x, y)$ and $s(x, y)=s_{k, l}(x, y)$ if $(x, y) \in X_{k} \times Y_{l}$. By their definition and Theorem 3.6, $s$ and $t$ belong to $\mathcal{V}(X, Y)$.

Conversely, suppose that $\varphi \sim t / s$ for some functions $t, s \in \mathcal{V}(X, Y)$ with $s(x, y) \neq 0$ for every $(x, y) \in X \times Y$. By Lemma 3.3, $X \times Y$ can be decomposed into a countable union of rectangles on each of which $t$ is a Schur multiplier. Applying the same lemma to each of these rectangles, we decompose $X \times Y$ into the union of rectangles $\kappa_{m}$ on each of which both $t$ and $s$ are Schur multipliers. By the $\sigma$-finiteness of the measure spaces, we may moreover assume that $(\mu \times \nu)\left(\kappa_{m}\right)<\infty$ for each $m \in \mathbb{N}$. It follows that $\left.s\right|_{\kappa_{m}}$ and $\left.t\right|_{\kappa_{m}}$ are equivalent to functions from $\Gamma\left(\kappa_{m}\right)$. An application of Lemma 4.3 now implies that $\varphi$ is a $\mathrm{w}^{*}$-closable multiplier.

Corollary 4.5. The set $\mathfrak{S}_{w^{*}}(X, Y)$ of all $w^{*}$-closable multipliers is a subalgebra of $\mathfrak{B}(X \times Y)$ which contains $\mathfrak{S}_{\text {loc }}(X, Y)$. Moreover, every $w^{*}$-closable multiplier $\varphi$ is equivalent to an $\omega$-continuous function.

Proof. The fact that the collection of all $\mathrm{w}^{*}$-closable multipliers is an algebra follows from Theorem 4.4 and Proposition 3.2. Theorems 3.6 and 4.4 imply that every local multiplier is $\mathrm{w}^{*}$-closable.

Let $\varphi \in \mathfrak{B}(X \times Y)$ be a w*-closable multiplier. By Theorem 4.3, $\varphi=\frac{t}{s}$ almost everywhere on $X \times Y$, where $s, t \in \mathcal{V}(X, Y)$. By Theorem 3.6, $t$ and $s$ are local multipliers hence they are $\omega$-continuous by Proposition 3.2.

It is easy to see that if $f$ is an $\omega$-continuous function and $g: \mathbb{C} \rightarrow \mathbb{C}$ is continuous on an open set containing $f(X \times Y)$ then $g \circ f$ is $\omega$-continuous. Hence $\frac{1}{s}$ is $\omega$-continuous, and since $\omega$-continuous functions form an algebra, $\frac{t}{s}$ is $\omega$-continuous. 
Let $\kappa_{\varphi}^{w^{*}} \subseteq X \times Y$ be the complement of the $\omega$-union of the family of all rectangles $\alpha \times \beta$ such that $\left.\varphi\right|_{\alpha \times \beta} \in \mathfrak{S}_{w^{*}}(\alpha, \beta)$. The next proposition will be useful for us in the subsequent sections.

Proposition 4.6. Let $\varphi \in \mathfrak{B}(X \times Y)$. Then $\kappa_{\varphi}^{w^{*}}=\operatorname{null} D\left(S_{\varphi}^{*}\right)$.

Proof. It follows from Lemma 2.3 that $\varphi$ is a $\mathrm{w}^{*}$-closable multiplier if and only if null $D\left(S_{\varphi}^{*}\right)=\emptyset$. Applying this to an arbitrary rectangle $\alpha \times \beta \subseteq X \times Y$ together with the observation that null $D\left(S_{\left.\varphi\right|_{\alpha \times \beta} ^{*}}\right)=(\alpha \times \beta) \cap$ null $D\left(S_{\varphi}^{*}\right)$, we obtain that $\alpha \times \beta$ has a marginally null intersection with null $D\left(S_{\varphi}^{*}\right)$ if and only if $\left.\varphi\right|_{\alpha \times \beta}$ is a $\mathrm{w}^{*}$-closable multiplier. This implies our statement.

It follows from Corollary 4.5 that $\kappa_{\varphi}^{w^{*}} \subseteq \kappa_{\varphi}$. It is natural to call the functions $\varphi \in \mathfrak{B}(X \times Y)$ for which $\kappa_{\varphi}^{w^{*}} \simeq X \times Y$ extremely non-w ${ }^{*}$-closable multipliers. We have that every extremely non-w*-closable multiplier is an extremely non-Schur multiplier.

\section{Closable multipliers}

In this section we study the class $\mathfrak{S}_{\mathrm{cl}}(X, Y)$ of closable multipliers. Let $\varphi \in$ $\mathfrak{B}(X \times Y)$. Recall that the transformation $S_{\varphi}$ is defined on the linear manifold $D(\varphi)=\left\{h \in L^{2}(X \times Y): \varphi h \in L^{2}(X \times Y)\right\}$ by letting $S_{\varphi} h=\varphi h$ and, after identifying $L^{2}(X \times Y)$ with $\mathcal{C}_{2}\left(H_{1}, H_{2}\right)$, is considered as a densely defined operator on the space $\mathcal{K}\left(H_{1}, H_{2}\right)$ of compact operators from $H_{1}$ into $H_{2}$. The dual space of $\mathcal{K}\left(H_{1}, H_{2}\right)$ is the space $\mathcal{C}_{1}\left(H_{2}, H_{1}\right)$ of nuclear operators; we identify it with $\Gamma(X, Y)$, and, by Lemma 4.2 , the domain of the adjoint operator is $D_{*}(\varphi) \stackrel{\text { def }}{=} D\left(S_{\varphi}^{*}\right)=\left\{h \in \Gamma(X, Y): \varphi h \in^{\mu \times \nu} \Gamma(X, Y)\right\}$. It follows from Proposition 2.6 that $\varphi \in \mathfrak{S}_{\mathrm{cl}}(X, Y)$ if and only if $D_{*}(\varphi)$ is weak ${ }^{*}$ dense in $\Gamma(X, Y)$. Equivalently, $\varphi \in \mathfrak{S}_{\mathrm{cl}}(X, Y)$ if and only if $D_{*}(\varphi)_{\perp}=0$, where $D_{*}(\varphi)_{\perp}$ is the set of all compact operators $K$ such that $\langle K, h\rangle=0$ for all $h \in D_{*}(\varphi)$. Note that $D_{*}(\varphi)$ is a sub-bimodule of the bimodule $\Gamma(X, Y)$ over the algebras $L^{\infty}(X, \mu)$ and $L^{\infty}(Y, \nu)$.

Let $D \subseteq \Gamma(X, Y)$ be any bimodule. Then, for all measurable sets $\alpha \subseteq X$, $\beta \subseteq Y$ and all $h \in D$, the function $\chi_{\alpha}(x) \chi_{\beta}(y) h(x, y)$ belongs to $D$. One can choose the sets $\alpha$ and $\beta$ in such a way that this function is a Schur multiplier. Indeed, if $h(x, y)=(a(x), b(y))$ for some square integrable Hilbert space valued functions $a$ and $b$, then it suffices to set $\alpha=\{x:\|a(x)\| \leq N\}$ and $\beta=\{y:\|b(y)\| \leq N\}$, for some $N>0$. Letting $N$ tend to infinity, we moreover see that $D \cap \mathfrak{S}(X, Y)$ is norm dense in $D$.

We will need the following proposition.

Proposition 5.1. Let $D_{1}, D_{2} \subseteq \Gamma(X, Y)$ be weak* dense bimodules, invariant under $\mathfrak{S}(X, Y)$. Then $D_{1} \cap D_{2}$ is weak* dense.

Proof. We identify the predual of $\Gamma(X, Y)$ with $\mathcal{K}\left(H_{1}, H_{2}\right)$. Let $K \in\left(D_{1} \cap\right.$ $\left.D_{2}\right)_{\perp}$ and $\theta_{i} \in D_{i} \cap \mathfrak{S}(X, Y), i=1,2$. By the invariance of $D_{1}$ and $D_{2}$ under $\mathfrak{S}(X, Y)$, we have that $\theta_{1} \theta_{2} \in D_{1} \cap D_{2}$. Thus, $\left\langle K, \theta_{1} \theta_{2}\right\rangle=0$ and therefore 
$\left\langle S_{\theta_{1}}(K), \theta_{2}\right\rangle=0$ for all $\theta_{2} \in D_{2} \cap \mathfrak{S}(X, Y)$. Since $D_{2} \cap \mathfrak{S}(X, Y)$ is dense in $D_{2}$ and $S_{\theta_{1}}(K)$ is a compact operator, we have that $S_{\theta_{1}}(K)=0$. Thus, $\left\langle K, \theta_{1}\right\rangle=0$ for all $\theta_{1} \in D_{1} \cap \mathfrak{S}(X, Y)$ and hence $K=0$.

Theorem 5.2. $\mathfrak{S}_{\mathrm{cl}}(X, Y)$ is a subalgebra of $\mathfrak{B}(X \times Y)$.

Proof. Let $\varphi_{1}$ and $\varphi_{2}$ be closable multipliers. By Theorem 2.5 and Lemma 4.2 (i), the bimodules $D_{*}\left(\varphi_{1}\right)$ and $D_{*}\left(\varphi_{2}\right)$ are invariant under $\mathfrak{S}(X, Y)$; moreover, $D_{*}\left(\varphi_{1}\right) \cap D_{*}\left(\varphi_{2}\right) \subseteq D_{*}\left(\varphi_{1}+\varphi_{2}\right)$. Propositions 5.1 and 2.6 imply that $\varphi_{1}+\varphi_{2}$ is closable.

To verify that $\mathfrak{S}_{\mathrm{cl}}(X, Y)$ is closed under products, it suffices now to show that if $\varphi$ is closable then $\varphi^{2}$ is closable. Let $D=D_{*}(\varphi)=\{h \in$ $\left.\Gamma(X, Y): \varphi h \in^{\mu \times \nu} \Gamma(X, Y)\right\}$ and $D_{0}=\left\{h \in \mathfrak{S}(X, Y) \cap \Gamma(X, Y): \varphi h \in^{\mu \times \nu}\right.$ $\mathfrak{S}(X, Y) \cap \Gamma(X, Y)\}$. Then $D_{0}$ is dense in $D$ and hence in $\Gamma(X, Y)$.

The product of a Schur multiplier and a closable multiplier is closable (indeed, if $w \in \mathfrak{S}(X, Y)$, then $D_{*}(\varphi) \subseteq D_{*}(w \varphi)$ whence $D_{*}(w \varphi)$ is dense). It follows that if $h \in D_{0}$ then $\psi \stackrel{\text { def }}{=} \varphi^{2} h=\varphi(\varphi h)$ is closable.

Fix $h \in D_{0}$ and let $k \in D_{*}(\psi)$. Then $h k \in D_{*}\left(\varphi^{2}\right)$. Hence, if $K \perp D_{*}\left(\varphi^{2}\right)$ then $0=(K, h k)=\left\langle S_{h}(K), k\right\rangle$. Since $D_{*}(\psi)$ is dense, we have that $\langle K, h\rangle=$ $S_{h}(K)=0$. Since $D_{0}$ is dense, $K=0$. Thus $D_{*}\left(\varphi^{2}\right)$ is dense and $\varphi^{2}$ is closable.

Following the analogy with harmonic analysis initiated in [1] and later pursued in [12], let us call an $\omega$-closed set $E \subseteq X \times Y$ an operator $M$-set (respectively, operator $M_{1}$-set) if $E$ supports a non-zero compact operator (resp. $\mathfrak{M}_{\min }(E)$ contains a non-zero compact operator). Clearly, every operator $M_{1}$-set is an operator $M$-set. We shall show in Section 7 that there exist operator $M$-sets which are not operator $M_{1}$-sets. We will shortly see that the property of being or not being an operator $M$ - (resp. $M_{1^{-}}$) set is important for deciding whether a given function is a closable multiplier. We hence include a consequence of Proposition 5.1 concerning sets which are not operator $M$ - or $M_{1}$-sets.

Proposition 5.3. Let $E_{1}, E_{2} \subseteq X \times Y$ be $\omega$-closed sets. Suppose that $E_{1}$ and $E_{2}$ are not operator $M$-sets (resp. not operator $M_{1}$-sets). Then $E_{1} \cup E_{2}$ is not an operator $M$-set (resp. not an operator $M_{1}$-set).

Proof. Suppose that $E_{1}$ and $E_{2}$ are not operator $M_{1}$-sets. Setting $D_{i}=$ $\mathfrak{M}_{\text {min }}\left(E_{i}\right)_{\perp}$, we have that $D_{i}$ is a weak* dense sub-bimodule of $\Gamma(X, Y)$, $i=1,2$. Note that, by [28], $D_{i}=\left\{\psi \in \Gamma(X, Y): \psi \chi_{E_{i}}=0\right.$ m.a.e. $\}, i=1,2$. It follows that $D_{i}$ is invariant under $\mathfrak{S}(X, Y), i=1,2$, and that

$$
D_{1} \cap D_{2}=\left\{\psi \in \Gamma(X, Y): \psi \chi_{E_{1} \cup E_{2}}=0 \text { m.a.e. }\right\} \text {. }
$$

By [28] again, $\left(D_{1} \cap D_{2}\right)^{\perp}=\mathfrak{M}_{\min }\left(E_{1} \cup E_{2}\right)$. By Proposition 5.1, $\left(D_{1} \cap D_{2}\right)^{\perp} \cap$ $\mathcal{K}\left(H_{1}, H_{2}\right)=\{0\}$ and hence $E_{1} \cup E_{2}$ is not an operator $M_{1}$-set.

Now suppose that $E_{1}$ and $E_{2}$ are not operator $M$-sets. Let $D_{i}=\left\{\psi \in \Gamma(X, Y): \psi\right.$ vanishes on an $\omega$-open neighbourhood of $\left.E_{i}\right\}$, 
$i=1,2$. By [28], $D_{i}^{\perp}=\mathfrak{M}_{\max }\left(E_{i}\right), i=1,2$. It is clear that $D_{1}$ and $D_{2}$ are invariant under $\mathfrak{S}(X, Y)$ and, since $E_{1}$ and $E_{2}$ are not operator $M$-sets, $D_{1}$ and $D_{2}$ are weak* dense in $\Gamma(X, Y)$. By Proposition 5.1, $D_{1} \cap D_{2}$ is weak* dense in $\Gamma(X, Y)$. However, $D_{1} \cap D_{2}$ equals

$\left\{\psi \in \Gamma(X, Y): \psi\right.$ vanishes on an $\omega$-open neighbourhood of $\left.E_{1} \cup E_{2}\right\}$

and hence $\left(D_{1} \cap D_{2}\right)^{\perp}=\mathfrak{M}_{\max }\left(E_{1} \cup E_{2}\right)$. Thus, $E_{1} \cup E_{2}$ is not an operator $M$-set.

In the next theorem, we relate the notions of operator $M$ - and operator $M_{1}$-sets to closability of multipliers.

Theorem 5.4. Let $\varphi \in \mathfrak{B}(X \times Y)$.

(i) If $\kappa_{\varphi}^{w^{*}}$ is not an operator $M$-set then $\varphi$ is a closable multiplier.

(ii) If $\kappa_{\varphi}^{w^{*}}$ is an operator $M_{1}$-set then $\varphi$ is not a closable multiplier.

Proof. It follows from Proposition 2.6 that $\varphi$ is closable if and only if $D\left(S_{\varphi}^{*}\right)^{\perp}$ $\cap \mathcal{K}\left(H_{1}, H_{2}\right)=\{0\}$. By [28] and Proposition 4.6 we have

$$
\mathfrak{M}_{\min }\left(\kappa_{\varphi}^{w^{*}}\right) \subseteq D\left(S_{\varphi}^{*}\right)^{\perp} \subseteq \mathfrak{M}_{\max }\left(\kappa_{\varphi}^{w^{*}}\right)
$$

which clearly implies the statement.

Corollary 5.5. (i) If $E$ is not an operator $M$-set and if, for every marginally disjoint from $E$ rectangle $\alpha \times \beta$, the restriction $\left.\varphi\right|_{\alpha \times \beta}$ is a $w^{*}$-closable multiplier, then $\varphi$ is a closable multiplier.

(ii) If $(\mu \times \nu)\left(\kappa_{\varphi}^{w^{*}}\right) \neq 0$ then $\varphi$ is not a closable multiplier.

Proof. (i) By the definition of $\kappa_{\varphi}^{w^{*}}$, we have that $\kappa_{\varphi}^{w^{*}} \subseteq E$, whence $\kappa_{\varphi}^{w^{*}}$ is not an operator $M$-set. The claim now follows from Theorem 5.4 (i).

(ii) Note that any set $E$ of non-zero measure is an operator $M_{1}$-set, because it supports a non-trivial Hilbert-Schmidt operator, and all such operators belong to $\mathfrak{M}_{\min }(E)$ [1]. So it suffices to apply Theorem 5.4 (ii).

Remark 5.6. (i) If the set $\kappa_{\varphi}^{w^{*}}$ is synthetic then $\varphi$ is a closable multiplier if and only if $\mathfrak{M}_{\max }\left(\kappa_{\varphi}^{w^{*}}\right)$ does not contain a non-zero compact operator.

(ii) Since $\kappa_{\varphi}^{w^{*}} \subseteq \kappa_{\varphi}$, we obtain that for the closability of $\varphi$ it suffices to show that $\kappa_{\varphi}$ does not support non-zero compact operators.

(iii) In Section 7 we shall construct a non-closable multiplier $\varphi$ such that $\kappa_{\varphi}^{w^{*}}$ is an operator $M$-set but not an operator $M_{1}$-set.

Example 5.7. Let $E \subseteq X \times Y$ be $\omega$-closed and let $\partial E$ be its $\omega$-boundary (that is, $\partial E=E \backslash E_{0}$, where $E_{0}$ is the largest, up to marginal equivalence, $\omega$-open set contained in $E[10])$. If $\varphi=\chi_{E}$ then for each rectangle $\alpha \times \beta$ marginally contained either in $E$ or in $E^{c}$, we have that $\left.\varphi\right|_{\alpha \times \beta}$ is a Schur multiplier and hence $\kappa_{\varphi}^{w^{*}}$ is marginally contained in $\partial E$. If $\partial E$ is not an operator $M$-set then, by Theorem 5.4, $\chi_{E}$ is a closable multiplier. 
We now present our first example of a non-closable multiplier, using a result on spectral (non)-synthesis.

Example 5.8. Let $U$ be the bilateral shift acting on the space $\ell^{2}(\mathbb{Z})$, that is, the operator given by $U e_{n}=e_{n+1}, n \in \mathbb{N}$, where $\left\{e_{n}\right\}_{n \in \mathbb{Z}}$ is the standard basis of $\ell^{2}(\mathbb{Z})$. Fix $p>2$. By [29, Proposition 9.9], there exist sequences $\left\{a_{n}\right\}_{n \in \mathbb{Z}},\left\{b_{n}\right\}_{n \in \mathbb{Z}} \in \ell^{2}(\mathbb{Z})$ with $\left|a_{n}\right|=\left|b_{n}\right|$, and an operator $X \in \mathcal{C}_{p}\left(\ell^{2}(\mathbb{Z})\right)$ such that

$$
\sum_{n \in \mathbb{Z}}\left(a_{n} U^{n}\right) X\left(b_{n} U^{-n}\right)=0 \text { and } \sum_{n \in \mathbb{Z}}\left(a_{n} U^{n}\right)^{*} X\left(b_{n} U^{-n}\right)^{*} \neq 0 .
$$

Let $W: \ell^{2}(\mathbb{Z}) \rightarrow L^{2}(\mathbb{T})$ be the inverse Fourier transform. Then $W U W^{*}$ is the operator of multiplication by $e^{i t}$ and $T=W X W^{*}$ is an operator in $\mathcal{C}_{p}\left(L^{2}(\mathbb{T})\right.$ ) satisfying

$$
\sum_{n \in \mathbb{Z}} M_{f_{n}} T M_{g_{n}}=0 \text { and } \sum_{n \in \mathbb{Z}} M_{\bar{f}_{n}} T M_{\bar{g}_{n}} \neq 0,
$$

where $M_{f_{n}}$ and $M_{g_{n}}$ are the multiplication operators by the functions $f_{n}$ and $g_{n}$ given by $f_{n}(t)=a_{n} e^{i n t}$ and $g_{n}(t)=b_{n} e^{-i n t}$, respectively. Set $d_{n}=a_{n} b_{n}$ and note that $\left\{d_{n}\right\} \in \ell^{1}(\mathbb{Z})$. Let $\psi(t, s)=\sum_{n \in \mathbb{Z}} f_{n}(t) g_{n}(s)=\sum_{n \in \mathbb{Z}} d_{n} e^{i n(t-s)}$. As $\sum_{n \in \mathbb{Z}}\left|f_{n}(t)\right|^{2}=\sum_{n \in \mathbb{Z}}\left|g_{n}(s)\right|^{2}=\sum_{n \in \mathbb{Z}}\left|d_{n}\right|<\infty$ for all $s, t \in \mathbb{T}$, Theorem 2.5 shows that the function $\psi$ is a Schur multiplier on $\mathbb{T} \times \mathbb{T}$ (equipped with the product Lebesgue measure). Let

$$
\varphi(t, s)= \begin{cases}\overline{\psi(t, s)} & \text { if } \psi(t, s) \neq 0 \\ 0 & \text { otherwise. }\end{cases}
$$

We claim that $\varphi$ is not closable. To see this, assume that $\left\{T_{n}\right\}_{n=1}^{\infty} \subseteq \mathcal{C}_{2}\left(L^{2}(\mathbb{T})\right)$ is a sequence with $T_{n} \rightarrow T$ in the operator norm. Then

$$
S_{\psi}\left(T_{n}\right) \rightarrow S_{\psi}(T)=\sum_{n \in \mathbb{Z}} M_{f_{n}} T M_{g_{n}}=0
$$

However,

$$
S_{\varphi}\left(S_{\psi}\left(T_{n}\right)\right)=S_{\bar{\psi}}\left(T_{n}\right) \rightarrow S_{\bar{\psi}}(T)=\sum_{n \in \mathbb{Z}} M_{\bar{f}_{n}} T M_{\bar{g}_{n}} \neq 0 .
$$

Example 5.8 will be considerably strengthened later: in Proposition 7.12, we will construct an $\omega$-continuous function which is a non-closable multiplier. On the other hand, the above example has the advantage that it exhibits a multiplier which is not closable in $\mathcal{C}_{p}$, for each $p>2$.

Let $[0,1]$ be the unit interval equipped with the Lebesgue measure, let $\Delta=\{(x, y) \in[0,1] \times[0,1]: x<y\}$ and $\varphi=\chi_{\Delta}$ be the characteristic function of $\Delta$. The multiplier $S_{\varphi}$ is usually called the transformer of triangular truncation (see for example [14]). The following result extends the well-known fact that $S_{\varphi}$ is not a Schur multiplier.

Proposition 5.9. The transformer of triangular truncation is closable but not $w^{*}$-closable. 
Proof. We first show that $\varphi$ is closable. Let $\Lambda=\{(x, x): x \in[0,1]\}$ be the diagonal of the unit square. The set $\Lambda$ only supports operators of multiplication by functions in $L^{\infty}(0,1)$; in particular, it is not an operator $M$-set. Since the function $\varphi$ is constant on each rectangle marginally disjoint from $\Lambda$, the claim follows from Corollary 5.5 (i).

To show that $\varphi$ is not $\mathrm{w}^{*}$-closable, it suffices, by Corollary 4.5, to show that $\varphi$ is not equivalent to an $\omega$-continuous function. Assume, towards a contradiction, that there exists an $\omega$-continuous function $f$ such that $f=\varphi$ almost everywhere. By Lemma 2.2, $f=0$ m.a.e. on $\Delta^{\prime}$ and $f=1$ m.a.e. on $\Delta$.

Note that if a rectangle is marginally disjoint from $\Delta$ or $\Delta^{\prime}$ then it is marginally disjoint from $\Lambda$. It follows that the same is true for any $\omega$-open set. Since $f^{-1}(\mathbb{C} \backslash\{1\})$ is marginally disjoint from $\Delta$, we obtain that $f=1$ m.a.e on $\Lambda$. Similarly $f=0$ m.a.e. on $\Lambda$. This is a contradiction because $\Lambda$ is not marginally null.

Remark The proof of Proposition 5.9 implies the following more general statement: Let $\Delta_{1}$ and $\Delta_{2}$ be disjoint $\omega$-open sets and $\Lambda=\left(\Delta_{1} \cup \Delta_{2}\right)^{c}$ be such that (a) $\Lambda$ does not support a non-zero compact operator, and (b) for every rectangle $\kappa, \kappa \cap \Lambda \not \varnothing \emptyset$ implies that $\kappa \cap \Delta_{i} \not \emptyset, i=1,2$. Then $\chi_{\Delta_{1}}$ is closable but not $\mathrm{w}^{*}$-closable.

Example 5.10. Let $E \subseteq X \times Y$ be an $\omega$-closed set such that $E \backslash \partial E \not \varnothing \emptyset$, where $\partial E$ is the $\omega$-boundary of $E$, and let $\varphi=\chi_{E}$. Then $k_{\varphi}^{w^{*}}=\operatorname{null} D\left(S_{\varphi}^{*}\right)=\partial E$.

In fact, if a rectangle $\kappa$ is such that $\kappa \cap \partial E \not \emptyset$ then, by the proof of Proposition $5.9,\left.\varphi\right|_{\kappa}$ is not $\omega$-continuous and hence not a $\mathrm{w}^{*}$-closable multiplier, giving that $\kappa$ is not marginally disjoint from $\kappa_{\varphi}^{w^{*}}$. As $\partial E$ marginally contains $\kappa_{\varphi}^{w^{*}}$ (see Example 5.7), we obtain $\kappa_{\varphi}^{w^{*}} \simeq \partial E$.

Proposition 5.9 shows that there exist closable multipliers which are not $\omega$-continuous. But they are continuous in the stronger pseudo-topology, $\tau$, introduced in Section 2.1.

Proposition 5.11. Any closable multiplier is $\tau$-continuous.

Proof. Let $\varphi \in \mathfrak{S}_{\mathrm{cl}}(X, Y)$. If $U \subseteq \mathbb{C}$ is an open set then

$$
f^{-1}(U)=\left(f^{-1}(U) \cap \kappa_{\varphi}^{w^{*}}\right) \cup\left(\left(f^{-1}(U) \cap\left(\kappa_{\varphi}^{w^{*}}\right)^{c}\right) .\right.
$$

Since $\kappa_{\varphi}^{w^{*}}$ is $\omega$-closed, $\left(\kappa_{\varphi}^{w^{*}}\right)^{c}$ is marginally equivalent to a countable union $\cup_{i=1}^{\infty} \alpha_{i} \times \beta_{i}$ of rectangles. Moreover, for each $i,\left.f\right|_{\alpha_{i} \times \beta_{i}}$ is $\mathrm{w}^{*}$-closable and hence $\omega$-continuous. This implies that $f^{-1}(U) \cap\left(\alpha_{i} \times \beta_{i}\right)$ is marginally equivalent to a countable union of rectangles and hence the same is true for $f^{-1}(U) \cap\left(\kappa_{\varphi}^{w^{*}}\right)^{c}$. It remains to note that $(\mu \times \nu)\left(\left(f^{-1}(U) \cap \kappa_{\varphi}^{w^{*}}\right)=0\right.$ because, by Corollary $5.5,(\mu \times \nu)\left(\kappa_{\varphi}^{w^{*}}\right)=0$.

Remark 5.12. We note that the class of $\tau$-continuous functions is strictly larger than that of closable multipliers; see Proposition 7.12. 


\section{Divided differences}

Let $f$ be a continuous function on a finite or infinite open subinterval $J \subseteq \mathbb{R}$. The divided difference of $f$ is the function

$$
\check{f}(x, y)=\frac{f(x)-f(y)}{x-y}
$$

defined on $J \times J \backslash \Lambda$, where $\Lambda=\{(x, x): x \in \mathbb{R}\}$. Let $\mu$ be a regular measure on $\mathbb{R}$ whose support contains $J$. In what follows we will assume that $\mu$ is non-atomic and hence $f$ is defined almost everywhere on $J \times J$.

The property of $\check{f}$ being a Schur multiplier is closely related to a kind of "operator smoothness" of $f$. Recall that $f$ is called operator Lipschitz (OL) on a compact subset $K \subseteq J$ if there exists a constant $D>0$ such that

$$
\|f(A)-f(B)\| \leq D\|A-B\|
$$

for all selfadjoint operators $A, B$ with spectra in $K$. The smallest constant $D$ with this property will be denoted by $|f|_{\mathrm{OL}}$.

Let $O(f)$ be the union of all open subintervals $I \subseteq J$ on which $f$ is OL. It is an open subset of $J$. Its complement will be denoted by $E(f)$.

Lemma 6.1. Let I be a compact subset of $J$. A function $\check{f}$ is a Schur multiplier on $I \times I$ if and only if $f$ is $O L$ on $I$.

Proof. If $\check{f}$ is a Schur multiplier then, for $h_{1}(x, y)=(x-y) h(x, y)$, we have

$$
\left\|I_{\check{f} h_{1}}\right\| \leq C\left\|I_{h_{1}}\right\|
$$

and hence

$$
\|f(A) X-X f(A)\| \leq C\|A X-X A\|,
$$

where $A$ is the operator of multiplication by $x$ on $L^{2}(I, \mu)$ and $X=I_{h}$. By $[22$, Remark 2.1, Corollary 3.6] and [23, Theorem 3.4], $f$ is OL.

Conversely, if $f$ is OL, then (2) holds for each $X \in \mathcal{B}\left(L^{2}(I, \mu)\right)$ by [22, Corollary 3.6]. This implies (1) for $L^{2}$-functions of the form $h_{1}(x, y)=$ $(x-y) h(x, y)$, where $h \in L^{2}(I \times I, \mu \times \mu)$. Since functions of this form are dense in $L^{2}(I \times I, \mu \times \mu)$, and since the $L^{2}$-norm majorizes the operator norm, inequality (1) holds for all $h_{1} \in L^{2}(I \times I, \mu \times \mu)$. This means that $\check{f}$ is a Schur multiplier.

Lemma 6.2. If $I_{1}, I_{2}$ are compact intervals and $I_{1} \cap I_{2}=\emptyset$ then $\left.\check{f}\right|_{I_{1} \times I_{2}} \in$ $\mathfrak{S}\left(I_{1}, I_{2}\right)$.

Proof. Since $f(x)-f(y) \in \mathfrak{S}\left(I_{1}, I_{2}\right)$, it suffices to show that $\left.\frac{1}{x-y}\right|_{I_{1} \times I_{2}} \in$ $\mathfrak{S}\left(I_{1}, I_{2}\right)$. Without loss of generality we may assume that $I_{1}=[0, a], I_{2}=[b, c]$ with $b>a$. We have

$$
\frac{1}{x-y}=-\sum_{n=0}^{\infty} \frac{x^{n}}{y^{n+1}}, \quad(x, y) \in I_{1} \times I_{2} .
$$

Since $\left\|\frac{x^{n}}{y^{n+1}}\right\|_{\mathfrak{S}\left(I_{1}, I_{2}\right)} \leq \frac{a^{n}}{b^{n+1}}$, the series converges in $\mathfrak{S}\left(I_{1}, I_{2}\right)$ in norm. 
The following theorem gives a precise description of the set of LMsingularity for a divided difference.

Theorem 6.3. For every continuous function $f$, we have

$$
\kappa_{\check{f}} \simeq\{(x, x): x \in E(f)\} .
$$

Proof. Write $O(f)$ as the union of a sequence of disjoint open intervals: $O(f)=\cup_{n=1}^{\infty} J_{n}$. For each $n, J_{n} \times J_{n}$ is the union of rectangles $I_{k} \times I_{k}$, where $I_{k}$ are compact subintervals of $J_{n}$. Since, by Lemma $6.1,\left.\check{f}\right|_{I_{k} \times I_{k}} \in \mathfrak{S}\left(I_{k}, I_{k}\right)$, it follows that $\left.\check{f}\right|_{J_{n} \times J_{n}} \in \mathfrak{S}_{\text {loc }}\left(J_{n}, J_{n}\right)$. Thus $\left(J_{n} \times J_{n}\right) \cap \kappa_{\check{f}} \simeq \emptyset$. Furthermore, $\kappa_{\breve{f}} \subseteq \Lambda$ by Lemma 6.2. It follows that, up to a marginally null set, we have

$$
\kappa_{\check{f}} \subseteq \Lambda \backslash\left(\cup_{n=1}^{\infty} J_{n} \times J_{n}\right)=\{(x, x): x \in E(f)\} .
$$

To prove the converse inclusion, it suffices to show by the regularity of $\mu$ that if $I_{1}$ and $I_{2}$ are compact subsets of $J$ such that $\left.\check{f}\right|_{I_{1} \times I_{2}} \in \mathfrak{S}\left(I_{1}, I_{2}\right)$ then $E(f) \cap I_{1} \cap I_{2}=\emptyset$; indeed, we would then have $\left(I_{1} \times I_{2}\right) \cap\{(x, x): x \in$ $E(f)\}=\left\{(x, x): x \in E(f) \cap I_{1} \cap I_{2}\right\} \simeq \emptyset$.

Let $I=I_{1} \cap I_{2}$. By Lemma 2.4 (i), $\left.\check{f}\right|_{I \times I} \in \mathfrak{S}(I, I)$, and Lemma 6.1 implies that $f$ is OL on $I$; therefore $I \subseteq O(f)$ and hence $I \cap E(f)=\emptyset$.

Corollary 6.4. $\check{f}$ is a local Schur multiplier if and only if $\mu(E(f))=0$.

It is known [21] that the class of all continuous Schur multipliers on $X \times Y$, where $X, Y$ are compact Hausdorff spaces, very weakly depends on the choice of Borel measures on $X$ and $Y$ : it depends only on the support of a measure. The above corollary shows that the class of continuous local Schur multipliers essentially depends on the choice of a measure. Indeed, a change of the measure does not change the set $E(f)$ while the condition $\mu(E(f))=0$ need not be preserved.

Corollary 6.5. For each $f$, the function $\check{f}$ is a closable Schur multiplier.

Proof. By Theorem 6.3, $\kappa_{\check{f}} \subseteq\{(x, x): x \in J\}$. Since the diagonal $\{(x, x)$ : $x \in J\}$ does not support a compact operator, it follows from Theorem 5.4 that $\check{f}$ is not closable.

Proposition 6.6. There exists a function $f:[0,1] \rightarrow \mathbb{C}$ such that $\check{f}$ is a Schur multiplier, $\check{f} \neq 0$ almost everywhere and $1 / \check{f}$ is not a local Schur multiplier.

Proof. Let $M$ be a Cantor-like set of non-zero Lebesgue measure (see [18]) and let $g$ be a continuously differentiable function which is equal to zero on $M$ and positive otherwise. Let $f$ be its primitive function: $f^{\prime}=g$. Then $f \in C^{2}([0,1])$ and hence it is operator Lipschitz [9]; by Lemma $6.1, \check{f}$ is a Schur multiplier. Since $f$ is strictly monotone, $\check{f} \neq 0$ almost everywhere.

The function $1 / \check{f}$ which, since $f$ is strictly monotone, is defined almost everywhere, is not a local Schur multiplier. In fact, assuming the converse, given $\epsilon>0$, we can find subsets $X_{\epsilon}, Y_{\epsilon}$ of $[0,1]$ such that $m\left([0,1] \backslash X_{\epsilon}\right)<\epsilon$, $m\left([0,1] \backslash Y_{\epsilon}\right)<\epsilon$ and $\left.(\check{f})^{-1}\right|_{X_{\epsilon} \times Y_{\epsilon}}$ is a Schur multiplier. Hence $(\check{f})^{-1}$ is equivalent to an essentially bounded function. But this is impossible since by 
construction $(\check{f})^{-1}(x, y)$ is arbitrary large for $(x, y)$ close to $(x, x), x \in M$ and since $m(M)>0$, the set $\left\{(x, y) \in X_{\epsilon} \times Y_{\epsilon}:\left|(\check{f}(x, y))^{-1}\right|>C\right\}$ has positive measure for all $C>0$ and sufficiently small $\epsilon>0$.

The divided difference $\check{f}$ can be extended to a continuous function on $J \times J$ if and only if $f$ is continuously differentiable. Our next aim is to construct a continuously differentiable function $f$ such that $\check{f}$ is not a Schur multiplier on each rectangle with non marginally null intersection with $\Lambda$. For this we need an extension of the well-known result of Farforovskaya [11] (see also Peller [26]) which states that a continuously differentiable function on a compact interval need not be OL.

Theorem 6.7. There is a function in $C^{1}([0,1])$ which is not $O L$ on each subinterval of $[0,1]$.

Proof. By [11], there exists $f \in C^{1}([0,1])$ which is not operator Lipschitz on $[0,1]$. Such function $f$ can be chosen so that

$$
f(0)=f^{\prime}(0)=f(1)=f^{\prime}(1)=0 .
$$

To see this it suffices to choose a continuously differentiable non OL function $g$ on a subinterval $I \subseteq(0,1)$ and extend it to a continuously differentiable function $f$ on $[0,1]$ satisfying $(3)$.

Let us denote by $C_{0}^{1}$ the set of all $f \in C^{1}([0,1])$ satisfying (3). Let $C_{s}=C^{\infty}([0,1]) \cap C_{0}^{1}$. It is well-known that all functions in $C^{\infty}$ are OL (in fact, it suffices for $f$ to have a continuous second derivative). We claim that for each $C>0$, there exists $g \in C_{s}$, such that $\|g\|_{C^{1}}=1$ and $|g|_{\mathrm{OL}}>C$.

Indeed suppose that this is not true. Since $C_{s}$ is dense in $C_{0}^{1}$, each function $f \in C_{0}^{1}$ is the sum of a series $\sum_{n=1}^{\infty} g_{n}$, where $g_{n} \in C_{s}$ and $\sum_{n=1}^{\infty}\left\|g_{n}\right\|_{C^{1}}<$ $\infty$. Our assumption gives $\sum_{n=1}^{\infty}\left|g_{n}\right|_{\mathrm{OL}}<\infty$ which easily implies that $|f|_{\mathrm{OL}}<$ $\infty$, and so $f \in O L$. This is a contradiction because, as we know from [11], $C_{0}^{1}$ is not contained in the set of all Operator Lipschitz functions.

Now, by [22], we may state that there exist operators $A=A^{*}$ and $X$ such that

$$
\|g(A) X-X g(A)\| \geq C / 2\|A X-X A\| .
$$

Moreover, by [22], $A$ and $X$ can be chosen to have finite rank. Clearly, the interval $[0,1]$ can be replaced by an arbitrary closed interval.

Let $\left\{I_{n}\right\}$ be a sequence of subintervals of $[0,1]$ such that each subinterval $J \subseteq[0,1]$ contains at least one (and hence infinitely many) $I_{n}$.

We claim that given operators of finite rank $X_{1}, \ldots, X_{n-1}, A_{1}, \ldots, A_{n-1}$, where $A_{i}^{*}=A_{i}, i=1, \ldots, n-1$, and a number $C>0$, there exist finite rank operators $A=A^{*}$ and $X$, and a smooth function $g$ such that $\operatorname{supp} g \subseteq I_{n}$, $\|g\|_{C^{1}([0,1])} \leq 1, g\left(A_{j}\right)=0, j=1, \ldots, n-1$, and $\|[g(A), X]\| \geq C\|[A, X]\|$.

Indeed, since the spectra of all $A_{j}$ are finite, one can find a subinterval $J$ of $I_{n}$ having empty intersection with $\cup_{j=1}^{n-1} \sigma\left(A_{j}\right)$. Now by the second paragraph, there exists a smooth function $g$ with support in $J$, such that $\|g\|_{\mathrm{OL}}>C$ and $\|g\|_{C^{1}}=1$. By the previous arguments this will imply the existence of operators $A$ and $X$ with the required properties. 
This allows us to construct sequences of operators $\left\{X_{n}\right\},\left\{A_{n}\right\}$, of smooth functions $\left\{g_{n}\right\}$ and of positive constants $\left\{C_{n}\right\}$ such that

1. $\left\|g_{n}\right\|_{C^{1}} \leq 1$

2. $\operatorname{supp} g_{n} \subseteq I_{n}$;

3. each $X_{n}, A_{n}$ are of finite rank and $A_{n}=A_{n}^{*}$;

4. $g_{n}\left(A_{j}\right)=0$ for $j<n$;

5. $\left\|\left[g_{n}\left(A_{n}\right), X_{n}\right]\right\| \geq C_{n}\left\|\left[A_{n}, X_{n}\right]\right\|$;

6. $C_{n} \geq 2^{n}\left(n+\sum_{j=1}^{n-1} 2^{-j}\left|g_{j}\right|_{\mathrm{OL}}\right)$.

Let $f(t)=\sum_{j=1}^{\infty} 2^{-j} g_{j}(t)$ so $f \in C^{1}([0,1])$. Let us prove that $f$ is not OL on any subinterval $J \subseteq[0,1]$. Assume the converse; then there exists $J \subset[0,1]$ and $C>0$ such that $\|[f(A), X]\| \leq C\|[A, X]\|$ for any $X$ and $A=A^{*}$ with $\sigma(A) \subseteq J$. By the choice of $I_{n}$, given $m>0$ there exists $n>m$ such that $I_{n} \subseteq J$. Therefore

$$
f\left(A_{n}\right)=\sum_{j=1}^{\infty} 2^{-j} g_{j}\left(A_{n}\right)=\sum_{j=1}^{n} 2^{-j} g_{j}\left(A_{n}\right) .
$$

Since $\left\|\left[f\left(A_{n}\right), X_{n}\right]\right\| \leq C\left\|\left[A_{n}, X_{n}\right]\right\|$, we have

$$
\begin{aligned}
& \left\|2^{-n}\left[g_{n}\left(A_{n}\right), X_{n}\right]\right\| \leq C\left\|\left[A_{n}, X_{n}\right]\right\|+\sum_{j=1}^{n-1} 2^{-j}\left\|\left[g_{j}\left(A_{n}\right), X_{n}\right]\right\| \\
& \leq\left(C+\sum_{j=1}^{n-1} 2^{-j}\left|g_{j}\right|_{\mathrm{OL}}\right)\left\|\left[A_{n}, X_{n}\right]\right\| .
\end{aligned}
$$

On the other hand,

$$
\left\|\left[g_{n}\left(A_{n}\right), X_{n}\right]\right\| \geq C_{n}\left\|\left[A_{n}, X_{n}\right]\right\|
$$

Hence

$$
C_{n} \leq 2^{n}\left(C+\sum_{j=1}^{n-1} 2^{-j}\left|g_{j}\right|_{\mathrm{OL}}\right)
$$

From condition (6) on the constant $C_{n}$ we get $2^{n}\left(n+\sum_{j=1}^{n-1} 2^{-j}\left\|g_{j}\right\|_{\mathrm{OL}}\right) \leq$ $2^{n}\left(C+\sum_{j=1}^{n-1} 2^{-j}\left\|g_{j}\right\|_{\mathrm{OL}}\right)$ and hence $n \leq C$ for every $n \in \mathbb{N}$, a contradiction.

Corollary 6.8. There exists $f \in C^{1}([0,1])$ such that $k_{\check{f}}=\{(x, x): x \in[0,1]\}$. Proof. Let $f$ be the function constructed in Theorem 6.7. Then $O(f)=\emptyset$ and $E(f)=[0,1]$. The statement now follows from Theorem 6.3.

\section{Multipliers of Toeplitz type}

Let $G$ be a locally compact second countable abelian group and therefore metrisable by $[17,8.3]$. Let $\mu=d s$ be the left invariant Haar measure on $G$. We write $L^{p}(G)$ for $L^{p}(G, \mu), p=1,2$, and denote by $C_{c}(G)$ the space of all continuous functions on $G$ with compact support. Let $\hat{G}$ be the dual group of 
$G$ and $A(G)$ (resp. $B(G)$ ) be the Fourier (resp. the Fourier-Stieltjes) algebra of $G$. We recall that $A(G)$ is the image of $L^{1}(\hat{G})$ under Fourier transform. It is well-known that $A(G)$ coincides with the family of functions $t \mapsto \int_{G} f(s-$ t) $g(s) d s=(\lambda(t) f, \bar{g}), f, g \in L^{2}(G)$, where $\lambda(t) f(s)=f(s-t)$. The algebra $B(G)$ is the image under Fourier transform of the convolution algebra $M(G)$ of all bounded Radon measures on $G$. One has $A(G) \subseteq B(G)$; equality holds if and only if $G$ is compact. It is moreover known that $B(G)$ coincides with the space of all multipliers $A(G)$ (see [31]).

For a subset $J \subseteq A(G)$, its null set is defined by

$$
\text { null } J=\{s \in G: f(s)=0 \text { for all } f \in J\} .
$$

Conversely, for a closed subset $E$ of $G$ we denote by $I(E)($ resp. $J(E))$ the space of all $f \in A(G)$ vanishing on $E$ (resp. the closed hull of the space of all $f \in A(G)$ vanishing on a neighborhood of $E)$; we have that $I(E)$ is the largest (resp. the smallest) closed ideal of $A(G)$ whose null set is equal to $E$ (see [31]).

Let $N$ be the map sending a measurable function $f: G \rightarrow \mathbb{C}$ to the function $N f: G \times G \rightarrow \mathbb{C}$ given by $N f(s, t)=f(s-t)$. The functions of the form $N f$ will be called functions of Toeplitz type. It is well-known (see, for example, [7]) that if $f \in L^{\infty}(G)$ then $N f$ is a Schur multiplier with respect to Haar measure if and only if $f \in^{\mu} B(G)$. In this section we show that the algebra of $\mathrm{w}^{*}$-closable multipliers of Toeplitz type coincides with that of local Schur multipliers of Toeplitz type; if $G$ is compact then both spaces coincide with the algebra of Schur multipliers of Toeplitz type, that is, with $N A(G)$.

We shall start with a result relating the continuity of a function $f$ on $G$ to the $\omega$-continuity of $N f$.

The following lemma is certainly known but, since we were not able to find a precise reference, we include its proof for completeness. Let $\mathcal{O}(X)$ denote the set of all open subset of a topological space $X$.

Lemma 7.1. Let $X$ be a topological space and $\xi: \mathcal{O}(\mathbb{C}) \rightarrow \mathcal{O}(X)$ be a union preserving map such that $\xi(\emptyset)=\emptyset, \xi(\mathbb{C})=X$ and $\xi(U \cap V)=\emptyset$ whenever $U, V \in \mathcal{O}(\mathbb{C})$ and $U \cap V=\emptyset$. Then there exists a continuous function $g$ : $X \rightarrow \mathbb{C}$ such that $\xi(U)=g^{-1}(U)$ for all $U \in \mathcal{O}(\mathbb{C})$.

Proof. For $t \in X$, let $O(t)$ denote the union of all $U \in \mathcal{O}(\mathbb{C})$ with $t \notin \xi(U)$. Since $\xi(\mathbb{C})=X$, we have that $\mathbb{C} \backslash O(t)$ is non-empty. If it contains at least two points, say $\lambda_{1}$ and $\lambda_{2}$, then taking disjoint open sets $U_{i}$ with $\lambda_{i} \in U_{i}$, $i=1,2$, we obtain that $t \in \xi\left(U_{1}\right) \cap \xi\left(U_{2}\right)$. This contradicts the fact that $\xi\left(U_{1}\right) \cap \xi\left(U_{2}\right)$ is empty.

We proved that $\mathbb{C} \backslash O(t)=\{\lambda\}$, for some $\lambda \in \mathbb{C}$. Setting $g(t)=\lambda$, we obtain a function $g: X \rightarrow \mathbb{C}$. It follows from its definition that $g^{-1}(U)=$ $\xi(U)$, for every $U \in \mathcal{O}(\mathbb{C})$. Hence, $g$ is continuous.

For $t \in G$, we denote by $\Lambda_{t}$ the $t$-shifted diagonal:

$$
\Lambda_{t}=\{(x, x-t): x \in G\} .
$$

We say that a subset $E$ of $\Lambda_{t}$ is non-null in $\Lambda_{t}$, if $m(\{x:(x, x-t) \in E\})>0$. 
For $W \subseteq C \times G$ set

$$
\pi(W)=\left\{t \in G: W \cap \Lambda_{t} \text { is non-null in } \Lambda_{t}\right\} .
$$

Clearly $\pi(G \times G)=G, \pi(\emptyset)=\emptyset$ and $\pi\left(W_{1} \cup W_{2}\right)=\pi\left(W_{1}\right) \cup \pi\left(W_{2}\right)$.

Lemma 7.2. If $W$ is $\omega$-open, then $\pi(W)$ is open.

Proof. Let $s \in \pi(W)$. It follows that there exists a rectangle $\alpha \times \beta \subseteq W$ with non-null (in $\Lambda_{s}$ ) intersection with $\Lambda_{s}$. By the $\sigma$-finiteness of the measure spaces, we may moreover assume that $\alpha$ and $\beta$ have finite measure. We now have $m(\alpha \cap(\beta+s))>0$. Since the function $x \rightarrow m(\alpha \cap(\beta+x))$ is continuous (being the convolution of the $L^{2}$-functions $\chi_{\alpha}$ and $\left.\chi_{\beta}\right), m(\alpha \cap(\beta+x))>0$ for all $x$ in a neighborhood $V$ of $s$. Hence $V \subseteq \pi(W)$.

Proposition 7.3. Let $f: G \rightarrow \mathbb{C}$ and $\varphi=N f$. The function $\varphi$ is equivalent to an $\omega$-continuous function if and only if $f$ is equivalent to a continuous function. Moreover, $\varphi$ is $\omega$-continuous if and only if $f$ is continuous.

Proof. If $f$ is continuous then $N f$ is continuous and hence $\omega$-continuous. It follows easily that if $f$ is equivalent to a continuous function then $N f$ is equivalent to a continuous function. We hence show the converse assertions.

Let $\psi: G \times G \rightarrow \mathbb{C}$ be an $\omega$-continuous function equivalent to $N f$. Thus, $Z \stackrel{\text { def }}{=}\{(x, y) \in G \times G: N f(x, y) \neq \psi(x, y)\}$ is a null set. Then $M \stackrel{\text { def }}{=} \pi(Z)$ is a null subset of $G$. Let us say that a point $t \in G$ is $\operatorname{good}$ if $t \notin M$.

For $U \in \mathcal{O}(\mathbb{C})$, set $\xi(U)=\pi\left(\psi^{-1}(U)\right)$. It follows from Lemma 7.2 that $\xi$ maps $\mathcal{O}(\mathbb{C})$ to $\mathcal{O}(G)$. The conditions $\xi(\mathbb{C})=G, \xi(\emptyset)=\emptyset$ and $\xi\left(U_{1} \cup U_{2}\right)=$ $\xi\left(U_{1}\right) \cup \xi\left(U_{2}\right)$ follow from the corresponding properties of $\pi$. We have to show that $\xi$ sends disjoint sets to disjoint sets.

Note that if $t$ is good and $t \in \xi(U)$ then $f(t) \in U$. Indeed, $\psi(x, x-t) \in U$ for all $x$ belonging to a certain non-null set, by the definition of $\xi(U)$. Since $t$ is good, for almost all $x \in G$, the pair $(x, x-t)$ does not belong to $Z$ hence there exists $x \in G$ such that $\psi(x, x-t)=N f(x, x-t)=f(t)$.

Now if $U_{1} \cap U_{2}=\emptyset$ and $\xi\left(U_{1}\right) \cap \xi\left(U_{2}\right) \neq \emptyset$ then $\xi\left(U_{1}\right) \cap \xi\left(U_{2}\right)$ is an open set must contain a good point $t \in G$. But then $f(t) \in U_{i}, 1=1,2$, a contradiction.

Applying Lemma 7.1 we obtain a continuous function $g: G \rightarrow \mathbb{C}$ with $\xi(U)=g^{-1}(U)$, for all $U \in \mathcal{O}(\mathbb{C})$. By the above argument, $g(t)=f(t)$ for all good $t$ (indeed for each $U$ containing $g(t)$ we have that $t \in g^{-1}(U)=\xi(U)$ whence $f(t) \in U$ ). Thus $f$ coincides almost everywhere with the continuous function $g$. $t \in G$.

If $N f$ is $\omega$-continuous then all points are good and $f(t)=g(t)$, for all

Let us say that a measurable function $f: G \rightarrow \mathbb{C}$ belongs (resp. almost belongs) to $A(G)$ at a point $t \in G$ if there exist a neighborhood $U$ of $t$ and a function $g \in A(G)$ such that $f(s)=g(s)$ everywhere (resp. almost everywhere with respect to Haar measure $\mu$ ) on $U$. If $f$ belongs to $A(G)$ at each $t \in G$ then we say that $f$ locally belongs to $A(G)$ and write $f \in A(G)^{\text {loc }}$. 
It is obvious that $A(G)^{\text {loc }} \subseteq C(G)$ and, using the regularity of $A(G)$, it is easy to show that if $G$ is compact then $A(G)^{\text {loc }}=A(G)$. In general we have the inclusions $A(G) \subseteq B(G) \subseteq A(G)^{\text {loc }}$.

If $f$ almost belongs to $A(G)$ at each point $t \in G$, it is not difficult to see that $f$ is equivalent to a function in $A(G)^{\text {loc }}$. We recall that in this case we write $f \in \in^{\mu} A(G)^{\text {loc }}$.

For a measurable function $f: G \rightarrow \mathbb{C}$, let

$$
J_{f}=\left\{h \in A(G): f h \in^{\mu} A(G)\right\}
$$

and $E_{f}=\operatorname{null} J_{f}$. Clearly, $J_{f}$ is an ideal of $A(G)$ whence

$$
J\left(E_{f}\right) \subseteq \overline{J_{f}} \subseteq I\left(E_{f}\right)
$$

Lemma 7.4. Let $f: G \rightarrow \mathbb{C}$ be measurable. Then

$$
E_{f}=\{t \in G: f \text { does not almost belong to } A(G) \text { at } t\} .
$$

Proof. Let $E$ be the set in the right hand side of (4). If $t \in E^{c}$, then $f$ almost belong to $A(G)$ at $t$ and therefore there exists a neighborhood $V$ of $t$ such that $f g$ is equivalent to a function in $A(G)$ for any $g \in A(G)$ with $V^{c} \subseteq$ null $\{g\}$. Now if $g \in A(G)$ takes the value 1 at $t$ and 0 on $V^{c}$ then $g \in J_{f}$ and $t \notin$ null $g$. Thus, $t \notin E_{f}$ and hence $E_{f} \subseteq E$.

To see the reverse inclusion, let $t \in E$ and assume that there exists $g \in A(G)$ such that $f g \sim h, h \in A(G)$ and $g(t) \neq 0$. Then there exists a neighborhood $U$ of $t$ such that $|g(s)|>\delta>0$ for all $s \in U$. By the regularity of $A(G)$, we can find $q \in A(G)$ such that $q(s) g(s)=1$ for all $s \in U$; therefore $f(s)=f(s) q(s) g(s)$ for all $s \in U$. Since $f g q \sim h q$ on $U$ and $h q \in A(G)$, the function $f$ almost belongs to $A(G)$ at $t$. We obtain a contradiction giving $E \subseteq E_{f}$

For notational simplicity we let $\Gamma(G)=\Gamma(G, G)$. We shall frequently use the map $P: \Gamma(G) \rightarrow A(G)$ given by $P(f \otimes g)(t)=(\lambda(t) g, \bar{f})$. Clearly, $P$ is a surjective contraction.

For a subset $E \subseteq G$, let $E^{*}=\{(x, y) \in G \times G: x-y \in E\}$.

Theorem 7.5. Assume that $G$ is a subgroup of $\mathbb{R}^{n}$ or $\mathbb{T}^{n}, n \in \mathbb{N}$. Let $f: G \rightarrow \mathbb{C}$ be a measurable function, $\varphi=N f$ and $U, V \subseteq G$ be measurable sets. The following are equivalent:

(i) $(U \times V) \cap E_{f}^{*} \simeq \emptyset$;

(ii) $\left.\varphi\right|_{U \times V} \in \mathfrak{S}_{\text {loc }}(U, V)$;

(iii) $\left.\varphi\right|_{U \times V} \in \mathfrak{S}_{w^{*}}(U, V)$.

Proof. Set $E=E_{f}$ and note that $(U \times V) \cap E^{*} \simeq \emptyset$ if and only if $\left(U^{\prime}-V^{\prime}\right) \cap E=$ $\emptyset$ for some $U^{\prime} \subseteq U$ and $V^{\prime} \subseteq V$ with $\mu\left(U \backslash U^{\prime}\right)=\mu\left(V \backslash V^{\prime}\right)=0$. We claim that $f \psi \in^{\mu} A(G)$ for every $\psi \in A(G) \cap C_{c}(G)$ with supp $\psi \subseteq E^{c}$. Indeed, by Lemma 7.4, for each $t \in E^{c}$ there exists a neighborhood $V_{t}$ of $t$ and a function $g_{t} \in A(G)$ such that $f \sim g_{t}$ on $V_{t}$. Since $\operatorname{supp} \psi$ is compact there exists a finite set $F \subseteq G$ such that $\operatorname{supp} \psi \subset \cup_{t \in F} V_{t}$. It follows from the regularity of $A(G)$ that there exist $h_{t} \in A(G), t \in F$, such that $\sum_{t \in F} h_{t}(x)=1$ if $x \in \operatorname{supp} \psi$ 
and $h_{s}(x)=0$ if $x \notin V_{s}$ for each $s \in F$ (see the proof of [17, Theorem 39.21]). Then for every $x \in G$ we have

$$
f(x) \psi(x)=\sum_{t \in F} f(x) \psi(x) h_{t}(x)
$$

and hence $f \psi \sim \sum_{t \in F} g_{t} \psi h_{t}$, giving $f \psi \in \in^{\mu} A(G)$.

(i) $\Rightarrow$ (ii) Suppose $(U \times V) \cap E^{*} \simeq \emptyset$ and let $U^{\prime} \subseteq U$ and $V^{\prime} \subseteq V$ be measurable subsets such that $m\left(U \backslash U^{\prime}\right)=m\left(V \backslash V^{\prime}\right)=0$ and $\left(U^{\prime}-V^{\prime}\right) \cap E=\emptyset$. Since $G$ is second countable and $U^{\prime}-V^{\prime} \subseteq E^{c}, m\left(U \backslash U^{\prime}\right)=m\left(V \backslash V^{\prime}\right)=0$, we may choose increasing sequences $\left\{K_{n}\right\}_{n=1}^{\infty}$ and $\left\{L_{n}\right\}_{n=1}^{\infty}$ of compact sets such that, up to a null set, $\cup_{n=1}^{\infty} K_{n}=U$ and $\cup_{n=1}^{\infty} L_{n}=V$, and a compact set $M_{n}$ such that $K_{n}-L_{n} \subseteq M_{n} \subseteq E^{c}$. Choose, for each $n \in \mathbb{N}$, a function $\psi_{n} \in$ $A(G) \cap C_{c}(G)$ supported in $E^{c}$ and taking value 1 on $M_{n}$. By the previous paragraph, $f \psi_{n} \in^{\mu} A(G)$ and therefore $N\left(f \psi_{n}\right)$ is a Schur multiplier. Thus, for each $\xi \in \Gamma(G)$, we have

$$
\varphi \chi_{K_{n} \times L_{n}} \xi=N\left(f \psi_{n}\right) \chi_{K_{n} \times L_{n}} \xi \in^{\mu \times \mu} \Gamma(G) .
$$

It follows that $\left.\varphi\right|_{K_{n} \times L_{n}}$ is a Schur multiplier and hence $\varphi \in \mathfrak{S}_{\text {loc }}(U, V)$.

(ii) $\Rightarrow$ (iii) follows from Corollary 4.5 .

(iii) $\Rightarrow$ (i) We will identify $\Gamma(U, V)$ with a subset of $\Gamma(G)$ in a natural way. Let $\psi=\left.\varphi\right|_{U \times V}$. By Proposition 2.6, $D\left(S_{\psi}^{*}\right)$ is norm dense in $\Gamma(U, V)$. Thus, $P\left(D\left(S_{\psi}^{*}\right)\right)$ is norm dense in $P(\Gamma(U, V))$. By Lemma $4.2(\mathrm{i}), D\left(S_{\psi}^{*}\right)=\{h \in$ $\left.\Gamma(U \times V): \psi h \in^{\mu \times \mu} \Gamma(U, V)\right\}$. Since $f P(h)=P(\varphi h) \in P(\Gamma(G)) \in^{\mu} A(G)$ for every $h \in D\left(S_{\psi}^{*}\right)$, the set $\left\{P(h): h \in \Gamma(U, V), f P(h) \in \in^{\mu} A(G)\right\}$ is dense in $P(\Gamma(U, V))$, and hence

$$
P(\Gamma(U, V)) \subseteq \overline{J_{f}}
$$

This implies that

$$
E=\operatorname{null} \overline{J_{f}} \subseteq \text { null } P(\Gamma(U, V)) .
$$

It suffices to show that there exist subsets $U^{\prime} \subseteq U, V^{\prime} \subseteq V$ such that $\mu\left(U \backslash U^{\prime}\right)=\mu\left(V \backslash V^{\prime}\right)=0$ and $\left(U^{\prime}-V^{\prime}\right) \cap$ null $P(\Gamma(U, V))=\emptyset$ since this will imply

$$
U^{\prime}-V^{\prime} \subseteq \operatorname{null} P(\Gamma(U, V))^{c} \subseteq E^{c}
$$

and hence $(U \times V) \cap E^{*} \simeq \emptyset$.

Let $U^{\prime}, V^{\prime}$ be the sets of density points of $U$ and $V$, respectively. Then, by the Lebesgue density theorem (see [25]), $\mu\left(U \backslash U^{\prime}\right)=\mu\left(V \backslash V^{\prime}\right)=0$. To prove the statement, it suffices to show that $P\left(\chi_{U} \otimes \chi_{V}\right)(s)=\mu(U \cap(V+$ $s))>0$ if $s \in U^{\prime}-V^{\prime}$. Let $s=u-v, u \in U^{\prime}, v \in V^{\prime}$ and assume that $\mu(U \cap(V+s))=\mu((U-u) \cap(V-v))=0$. If $B_{\epsilon}(0)$ is the closed ball with centre 0 and radius $\epsilon$, we then have

$$
\mu\left((U-u) \cap B_{\epsilon}(0)\right)+\mu\left((V-v) \cap B_{\epsilon}(0)\right) \leq \mu\left(B_{\epsilon}(0)\right),
$$

for each $\epsilon>0$. Applying the Lebesgue density theorem we obtain

$$
2=1+1=\lim _{\epsilon \rightarrow 0} \frac{\mu\left((U-u) \cap B_{\epsilon}(0)\right)}{\mu\left(B_{\epsilon}(0)\right)}+\lim _{\epsilon \rightarrow 0} \frac{\mu\left((V-v) \cap B_{\epsilon}(0)\right)}{\mu\left(B_{\epsilon}(0)\right)} \leq 1,
$$

a contradiction. 
Remark 7.6. (i) The condition that $G$ be a subgroup of $\mathbb{R}^{n}$ or $\mathbb{T}^{n}$ is only used to prove the implication (iii) $\Rightarrow(\mathrm{i}$ ), where we appeal to the Lebesgue density theorem. The statement remains true for more general groups (in particular, for Lie groups), for which there is an analog of the Lebesgue theorem (see $[8,24])$.

(ii) Taking $U=V=G$, we see that $\varphi \in \mathfrak{S}_{w^{*}}(G, G)$ implies $E_{f}=\emptyset$ for any group $G$, since in this case the arguments in the proof of Theorem 7.5 give $\overline{J_{f}}=P(\Gamma(G))=A(G)$.

We note some consequences of Theorem 7.5. In the next corollary, which gives a precise description of the sets $\kappa_{\varphi}$ and $\kappa_{\varphi}^{w^{*}}$, we assume that $G$ satisfies the conditions of Theorem 7.5 (see also Remark 7.6 (i)).

Corollary 7.7. Let $f: G \rightarrow \mathbb{C}$ be a measurable function and $\varphi=N f$. Then $\kappa_{\varphi} \simeq \kappa_{\varphi}^{w^{*}} \simeq\left(E_{f}\right)^{*}$.

The following theorem shows that the set of local Schur multipliers and that of $\mathrm{w}^{*}$-closable multipliers coincide in the class of Toeplitz functions.

Theorem 7.8. Let $G$ be an arbitrary second countable locally compact abelian group. Let $f: G \rightarrow \mathbb{C}$ be a measurable function and $\varphi=N f$. The following are equivalent:

(i) $f \in \in^{\mu} A(G)^{\mathrm{loc}}$;

(ii) $\varphi$ is a local Schur multiplier;

(iii) $\varphi$ is a $w^{*}$-closable multiplier.

If $G$ is compact then the above statements are equivalent to

(iv) $\varphi$ is a Schur multiplier.

Proof. We note that $f \in^{\mu} A(G)^{\text {loc }}$ if and only if $E_{f}=\emptyset$. The equivalence (i) $\Leftrightarrow($ ii $) \Leftrightarrow$ (iii) follows from Theorem 7.5 and Remark 7.6(ii).

Assume that $G$ is compact. Then (i) $\Leftrightarrow($ iv) follows from the equality $A(G)=A(G)^{\text {loc }}$ and the fact that $N f$ is a Schur multiplier if and only if $f \in \in^{m} A(G)$.

Our next result shows that the class of $\omega$-continuous functions is strictly larger than the class of $\mathrm{w}^{*}$-closable multipliers.

Corollary 7.9. Let $G$ be a compact abelian group, $f \in C(G) \backslash A(G)$ and $\varphi=N f$. Then $\varphi$ is $\omega$-continuous but not $w^{*}$-closable.

Proof. Since $\varphi$ is the composition of the continuous function $g$, given by $g(s, t)=s-t$, and the continuous function $f$, it is $\omega$-continuous. By Theorem $7.8, \varphi$ is not a $\mathrm{w}^{*}$-closable multiplier.

Example 7.10. Let $\Delta=\{(x, y) \in \mathbb{R}: x \leq y\}$. Then $\chi_{\Delta}(x, y)=\chi_{(-\infty, 0]}(x-y)$ is not a $\mathrm{w}^{*}$-closable multiplier, since $\chi_{(-\infty, 0]}$ does not almost belong to $A(\mathbb{R})$ at $x=0$. 
Remark 7.11. It is known that there exists a function $f \in B(\mathbb{R})$ such that $f>0$ on $\mathbb{R}$, but $1 / f \notin B(\mathbb{R})([13, \S 32])$. Since a function of Toeplitz type $w(s, t)=f(s-t), s, t \in \mathbb{R}, f \in C(\mathbb{R})$, is a Schur multiplier if and only if $f \in$ $B(\mathbb{R})$, we obtain a positive Schur multiplier $w$ such that $1 / w$ is not a Schur multiplier. However, $1 / w$ is a local Schur multiplier, since $1 / f \in A(\mathbb{R})^{\text {loc }}$. To see this, we note that $f$ belongs locally to $A(\mathbb{R})$ and hence for each $s \in \mathbb{R}$ there exists a neighbourhood $V_{s}$ and $g \in A(\mathbb{R})$ such that $f=g$ on $V_{s}$. Since $g(s) \neq 0$ on $V_{s}$, using the regularity of $A(\mathbb{R})$ one can find $h \in A(\mathbb{R})$ such that $h g=1$ on $V_{s}$. As $h=1 / f$ on $V_{s}$ and $s$ is arbitrary, we have $1 / f \in A(G)^{\text {loc }}$.

Note that, by Proposition 6.6, there exists a Schur multiplier $w$ such that $w(s, t) \neq 0$ almost everywhere and $1 / w$ is not a local Schur multiplier.

Proposition 7.12. There exists an $\omega$-continuous non-closable extremely-nonSchur multiplier.

Proof. Since each continuous function on $G \times G$ is $\omega$-continuous with respect to the Haar measure, it suffices to exhibit a continuous function $f$ such that $N f$ is non-closable and $\kappa_{f}=G \times G$. Let $G=\mathbb{T}$. By [20, Chapter II, Theorem 3.4], for any set $S \subseteq \mathbb{T}$ of Lebesgue measure zero there exists a function $h \in C(\mathbb{T})$ whose Fourier series diverges at every point of $S$. We can choose $S$ so that its closure is $\mathbb{T}$ and take the corresponding $f \in C(\mathbb{T})$. Let $\varphi=N f$. By the Riemann Localisation Lemma, any function which belongs to $A(\mathbb{T})$ at $x \in \mathbb{T}$ has a convergent Fourier series at $x$. Thus, $\mathbb{T} \subseteq E_{f}$ and hence $E_{f}=\mathbb{T}$.

By Corollary 7.7, we have $\kappa_{\varphi} \simeq \kappa_{\varphi}^{w^{*}} \simeq \mathbb{T}^{2}$ and therefore $\varphi$ is extremellynon-Schur multiplier. Moreover, applying now Proposition 4.6, we obtain null $D\left(S_{\varphi}^{*}\right)=\mathbb{T}^{2}$ and hence $D\left(S_{\varphi}^{*}\right)=\{0\}$, showing that $\varphi$ is non-closable.

Now assume $G$ is compact, so that $\hat{G}$ is discrete. Then $A(G)=\left\{\sum_{\chi \in \Gamma} c_{\chi} \chi\right.$ : $\left.\sum_{\chi \in \Gamma}\left|c_{\chi}\right|<\infty\right\}$. The space of pseudomeasures $P M(G)=A(G)^{*}$ can be identified with $\ell^{\infty}(\hat{G})$ via Fourier transform: $F \rightarrow\{\hat{F}(\chi)\}_{\chi \in \Gamma}$. A pseudomeasure $F \in P M(G)$ is called a pseudofunction if $\hat{F}$ vanishes at infinity. We recall that $P M(G)$ is an $A(G)$-module with respect to the operation $f F(g)=F(f g)$, for $F \in P M(G), f, g \in A(G)$, and that the support supp $F$ of a pseudomeasure $F$ is the set $\{x \in G: f F \neq 0$ whenever $f(x) \neq 0, f \in A(G)\}$.

If $E$ is a closed subset of $G$ we let $P M(E)$ (resp. $N(E)$ ) denote the space of all pesudomeasures supported in $E$ (resp. the weak* closed hull of the space of all measures $\mu \in M(G)$ supported in $E)$. Here, by the weak* topology we mean the $\sigma(P M(G), A(G))$-topology. Clearly, $N(E) \subseteq P M(E)$. Moreover, $P M(E)$ (resp. $N(E)$ ) is the largest (resp. smallest) weak* closed subspace the support of whose every element is in $E$. Moreover, $N(E)=I(E)^{\perp}$ and $P M(E)=J(E)^{\perp}$.

Recall that a closed set $E \subseteq G$ is called an $M$-set (resp. an $M_{1}$-set) if $P M(E)$ (resp. $N(E)$ ) contains a non-zero pseudofunction. It is known that there exists an $M$-set which is not an $M_{1}$-set, see [15, Section 4.6]. In what follows we shall give some sufficient conditions for a function of Toeplitz type to be a closable or a non-closable multiplier, based on the above notions. 
In [12], Froelich studied the question of when a given closed set supports a non-zero compact operator and a non-zero pseudo-integral compact operators. The next result uses ideas from [12]. We will use the fact that the restriction of the map $N$ (given by $N f(x, y)=f(x-y)$ ) to $A(G)$ takes values in the Varopoulos algebra $V(G) \stackrel{\text { def }}{=} C(G) \hat{\otimes} C(G) \subseteq \Gamma(G)$.

We will need a modification of the module action of $L^{1}(G)$ on $V(G)$ described on p. 365 of [30]. For $\psi \in \Gamma(G)$ and $r \in G$, write $r \cdot \psi$ for the function given by $r \cdot \psi(s, t)=\psi(s+r, t+r)$. If $f \in C(G)$, let $f \cdot \psi=\int_{G}(r$. $\psi) f(r) d r$, where the integral is understood in the Bochner sense. Following the arguments in [30], one can show that the action on $C(G)$ on $\Gamma(G)$ extends to an action of $L^{1}(G)$ on $\Gamma(G)$ and that if $\left\{f_{\alpha}\right\}_{\alpha}$ is a bounded approximate identity for $L^{1}(G)$ then $f_{\alpha} \cdot \psi \rightarrow \psi$ for every $\psi \in \Gamma(G)$.

The following lemma establishes that $E$ is an $M_{1}$-set if and only if $E^{*}$ is an operator $M_{1}$-set. This justifies the terminology introduced in Section 5 .

Lemma 7.13. Let $E \subseteq G$ be a closed set. The space $\mathfrak{M}_{\min }\left(E^{*}\right)$ contains a non-zero compact operator if and only if $E$ is an $M_{1}$-set.

Proof. Let $K$ be a non-zero compact operator supported on $E^{*}$. Then there exist $\gamma, \delta \in \hat{G}$ such that $c_{\delta, \gamma} \stackrel{\text { def }}{=}(K \gamma, \delta) \neq 0$. Let $F$ be the pseudomeasure given by

$$
\hat{F}(\chi)=c_{\gamma-\delta+\chi, \chi}, \quad \chi \in \hat{G} .
$$

Since $K$ is compact, $\hat{F}$ is a pseudo-function. For each $v=\sum_{\chi \in \hat{G}} a_{\chi} \chi \in I(E)$ (the sum being absolutely convergent), we have

$$
\begin{aligned}
F(v) & =\sum_{\chi \in \hat{G}} a_{\chi} \hat{F}(\chi)=\sum_{\chi} a_{\chi}(K \chi, \gamma-\delta+\chi) \\
& =\left\langle K, \sum_{\chi \in \hat{G}} a_{\chi} \chi \overline{\chi(\gamma-\delta)}\right\rangle=\langle K, \tilde{v}\rangle,
\end{aligned}
$$

where $\langle\cdot, \cdot\rangle$ is the duality between $\mathcal{B}\left(L^{2}(G)\right)$ and $\Gamma(G)$ and $\tilde{v}$ is the function given by $\tilde{v}(s, t)=N v(s, t) \overline{(\gamma-\delta)(t)}$. Since $N v$ vanishes on $E^{*}$ and $K \in$ $\mathfrak{M}_{\text {min }}\left(E^{*}\right)$, we have that $F(v)=0$, showing that $F$ is a pseudofunction in $N(E)$. Thus $E$ is an $M_{1}$-set.

Conversely, assume that $E$ is an $M_{1}$-set and let $F$ be a non-zero pseudofunction in $N(E)$. We let $K$ be the operator on $L^{2}(G)$ defined by $K \chi=\hat{F}(\chi) \chi$ on the orthonormal basis $\hat{G}$ of $L^{2}(G)$. Then for $v \in I(E)$, we have

$$
\langle K, N v\rangle=F(v)=0 .
$$

Suppose that $\psi \in \Gamma(G)$ vanishes marginally almost everywhere on $E^{*}$. For $\chi \in \hat{G}$, define the functions $\psi^{\chi}$ and $\tilde{\psi}^{\chi}$ by

$$
\psi^{\chi}(s, t)=\chi \cdot \psi(s, t) \text { and } \tilde{\psi}^{\chi}(s, t)=\chi(s) \psi^{\chi}(s, t) .
$$

We have that $\psi^{\chi}, \tilde{\psi}^{\chi} \in \Gamma(G)$, and $\tilde{\psi}^{\chi}(s+r, t+r)=\tilde{\psi}^{\chi}(s, t)$ marginally almost everywhere, for each $r \in G$ (see [30, Theorems 3.1 and 4.6]). Therefore, by [30, Proposition 4.5], $\tilde{\psi}^{\chi} \in N A(G)$. Since $\tilde{\psi}^{\chi}$ vanishes on $E^{*}, \tilde{\psi}^{\chi}=N v$ 
for some $v$ vanishing on $E$. By the previous paragraph, $\left\langle K, \tilde{\psi}^{\chi}\right\rangle=0$. This implies that $\left\langle K, \psi^{\chi}\right\rangle=0$. In fact, if $\chi \equiv 1$, this is trivial; if $\chi \not \equiv 1$ we have $\psi^{\chi}(s, t)=\chi(-s) \tilde{\psi}^{\chi}(t, s)=\chi(-s) v^{\chi}(s-t)$ for some $v^{\chi} \in A(G)$ and then writing $v^{\chi}(s)=\sum_{\tau \in \hat{G}} a_{\tau} \tau(s)$ and taking into account that $(\bar{\chi} \tau, \tau)=0$ for all $\tau \in \hat{G}$, we obtain

$$
\left\langle K, \psi^{\chi}\right\rangle=\sum_{\tau \in \hat{G}} a_{\tau}(K \bar{\chi} \tau, \tau)=\sum_{\tau \in \hat{G}} a_{\tau} F(\bar{\chi} \tau)(\bar{\chi} \tau, \tau)=0 .
$$

Finally, we let $\left\{u_{\alpha}\right\}$ be a bounded approximate identity for $L^{1}(G)$ chosen from $\operatorname{span}\{\chi: \chi \in \hat{G}\}$. For each $\alpha$, we have that $u_{\alpha} \cdot \psi \in \operatorname{span}\left\{\psi^{\chi}: \chi \in \hat{G}\right\}$ and hence $\left\langle K, u_{\alpha} \cdot \psi\right\rangle=0$ giving $\langle K, \psi\rangle=0$. Since $\psi$ is an arbitrary element of $\Gamma(G)$ vanishing on $E^{*}$, we conclude that $K \in \mathfrak{M}_{\min }\left(E^{*}\right)$.

Proposition 7.14. Let $f: G \rightarrow \mathbb{C}$ be a measurable function and $\varphi=N f$. Then the following holds:

(i) If $E_{f}$ is not an $M$-set then $\varphi$ is closable.

(ii) If $E_{f}$ is an $M_{1}$-set then $\varphi$ is not closable.

Proof. (i) By [12, Theorem 1.2.7, Lemma 1.2.10], $\mathfrak{M}_{\max }\left(E_{f}^{*}\right)$ does not contain a non-zero compact operator. The statement now follows from Theorem 5.4 (i) and Corollary 7.7.

(ii) follows from Theorem 5.4 (ii), Corollary 7.7 and Lemma 7.13.

Remark 7.15. We note that if $E_{f}$ satisfies spectral synthesis then $\varphi$ is closable if and only if $E_{f}$ is an $M$-set.

We say that a subset $E \subseteq G$ is $\tau_{0}$-open if $E$ is equivalent, with respect to the Haar measure, to an open subset of $G$. A function $f: G \rightarrow \mathbb{C}$ is said to be $\tau_{0}$-continuous if $f^{-1}(U)$ is $\tau_{0}$-open for any open $U \subseteq G$.

Proposition 7.16. Let $f: G \rightarrow \mathbb{C}$ be a measurable function and $\varphi=N f$. If $\varphi$ is closable then $f$ is $\tau_{0}$-continuous.

Proof. Since $f$ almost belongs to $A(G)$ at each point $t \in E_{f}^{c}$, it is equivalent to a continuous function $h$ on $E_{f}^{c}$. In fact, for each $t \in E_{f}^{c}$ there exists a neighborhood $U_{t}$ and $h_{t} \in A(G)$ such that $f=h_{t}$ almost everywhere on $U_{t}$. Since $G$ is second countable there exists a countable number of neighborhoods $U_{t_{i}}$ such that $E_{f}^{c}=\cup_{i=1}^{\infty} U_{t_{i}}$. Now set $h(t)=h_{t_{i}}(t)$ for $t \in U_{t_{i}}$. Clearly, $h$ is continuous on $E_{f}^{c}$ and $f=h$ a.e. on $E_{f}^{c}$. Thus, given an open subset $U \subseteq G$, the set $f^{-1}(U) \cap E_{f}^{c}$ is equivalent to an open set in $G$. Since

$$
f^{-1}(U)=\left(f^{-1}(U) \cap E_{f}^{c}\right) \cup\left(f^{-1}(U) \cap E_{f}\right),
$$

it is enough to show that $\mu\left(E_{f}\right)=0$.

Assume, by way of contradiction, that $\mu\left(E_{f}\right)>0$. Since $G$ is a compact group, $\chi_{E_{f}} \in L_{1}(G), \hat{\chi}_{E_{f}}$ vanishes at infinity and hence $\chi_{E_{f}}$ is a non-zero pseudo-function supported in $E_{f}$. But since, by Proposition $7.14, E_{f}$ is not an $M_{1}$-set, we arrive at a contradiction. 
We will finish this section by constructing an example of a non-closable multiplier $\varphi$ for which $\kappa_{\varphi}^{w^{*}}$ is an operator $M$-set but not an operator $M_{1}$-set. The existence of a closable multiplier for which $\kappa_{\varphi}^{w^{*}}$ is an operator $M$-set but not an operator $M_{1}$-set remains an open problem.

Example 7.17. Let $E \subseteq \mathbb{T}$ be s an $M$-set which is not an $M_{1}$-set. Then $\partial E=E$. We have that $\mathfrak{M}_{\min }\left(E^{*}\right)$ does not contain a non-zero compact operator, while $\mathfrak{M}_{\max }\left(E^{*}\right)$ contains such an operator, say $K$. .

As $\mathfrak{M}_{\max }\left(E^{*}\right) \neq \mathfrak{M}_{\min }\left(E^{*}\right)$, we can find $\Psi \in \Gamma(\mathbb{T})$ which vanishes on $E^{*}$ such that $\langle K, \Psi\rangle \neq 0$.

Let $\Psi_{1}=\sum_{n} \frac{1}{2^{n}} \chi_{\alpha_{n}} \otimes \chi_{\beta_{n}}$, where $\left\{\alpha_{n} \times \beta_{n}\right\}$ is a disjoint family of rectangles such that $\left(E^{*}\right)^{c} \simeq \cup_{n} \alpha_{n} \times \beta_{n}$. Then $\Psi_{1}$ is the limit of elements of $\Gamma(\mathbb{T})$ which vanish on an $\omega$-open subset of $\mathbb{T} \times \mathbb{T}$ containing $E^{*}$, and hence $\left\langle K, \Psi_{1}\right\rangle=0[28]$. We note that, moreover, null $\Psi_{1}=E^{*}$.

Now let

$$
\varphi(x, y)=\left\{\begin{array}{cl}
\frac{\Psi(x, y)}{\Psi_{1}(x, y)} & (x, y) \in\left(E^{*}\right)^{c} \\
0, & (x, y) \in E^{*} .
\end{array}\right.
$$

As $\Psi_{1} \in \Gamma(\mathbb{T})$, one can find measurable subsets $K_{n}, n \in \mathbb{N}$, with $K_{n} \subseteq K_{n+1}, n \in \mathbb{N}$, such that $m\left(K_{n}^{c}\right) \rightarrow_{n \rightarrow \infty} 0$ and $\Psi_{1} \chi_{K_{n} \times K_{n}}, \Psi \chi_{K_{n} \times K_{n}} \in$ $\mathfrak{S}\left(K_{n}, K_{n}\right), n \in \mathbb{N}$. Then there exists $N$ such that $\left\langle K, \Psi \chi_{K_{N} \times K_{N}}\right\rangle \neq 0$. On the other hand, we have $S_{\Psi_{1} \chi_{K_{N} \times K_{N}}}(K)=0$. Let $T_{n} \in \mathcal{C}_{2}\left(L^{2}(\mathbb{T})\right), T_{n} \rightarrow K$, $n \rightarrow \infty$. Then $S_{n} \stackrel{\text { def }}{=} M_{\chi_{K_{N}}} T_{n} M_{\chi_{K_{N}}} \rightarrow M_{\chi_{K_{N}}} K M_{\chi_{K_{N}}}$ and

$$
S_{\Psi_{1}}\left(S_{n}\right)=S_{\Psi_{1} \chi_{K_{N} \times K_{N}}}\left(S_{n}\right) \rightarrow S_{\Psi_{1} \chi_{K_{N} \times K_{N}}}(K)=0
$$

but

$$
S_{\varphi}\left(S_{\Psi_{1}}\left(S_{n}\right)\right)=S_{\Psi}\left(S_{n}\right) \rightarrow S_{\Psi}\left(M_{\chi_{K_{N}}} K M_{\chi_{K_{N}}}\right) \neq 0 .
$$

Thus $\varphi$ is not closable and hence $\kappa_{\varphi}^{w^{*}}$ is an operator $M$-set. As $\kappa_{\varphi}^{w^{*}} \subseteq E^{*}$, we have $\mathfrak{M}_{\min }\left(\kappa_{\varphi}^{w^{*}}\right) \subseteq \mathfrak{M}_{\min }\left(E^{*}\right)$ and hence $\kappa_{\varphi}^{w^{*}}$ is not an operator $M_{1}$-set.

\section{Open problems}

In this section we list some open problems. The most important question which we have left unanswered is the following:

Problem 1. Is every $\mathrm{w}^{*}$-closable multiplier a local Schur multiplier?

Problem 2. Does Theorem 7.5 hold for all locally compact abelian groups?

Problem 3. For which $f \in C(\mathbb{R})$ is the divided difference $\check{f}$ a $\mathrm{w}^{*}$-closable multiplier?

Since all local multipliers are $\mathrm{w}^{*}$-closable, Corollary 6.4 shows that a sufficient condition for this to happen is $\mu(E(f))=0$.

The last two problems are related to Problem 1. 
Problem 4. Let $f$ be an Operator Lipschitz function on $[a, b]$, and let $f^{\prime}(x) \neq 0$ for all $x \in[a, b]$. Is the inverse function $f^{-1}$ Operator Lipschitz on $[f(a), f(b)]$ ?

Problem 5. For which continuous functions $f$ and normal operators $A \in \mathcal{B}(H)$ is the map on $\mathcal{B}(H)$ given by $A X-X A \rightarrow f(A) X-X f(A)$ closable in weak* topology?

Remark 8.1. (i) It is natural to pose a question, similar to one in Problem 4, in Schatten-von Neumann ideals $\mathcal{C}_{p}$. As was proved by Potapov and Sukochev, [27], a function $f$ is (scalar) Lipschitz if and only if it is operator Lipschitz in $\mathcal{C}_{p}, 1<p<\infty$. Therefore, the answer to Problem 4 is negative if the operator Lipschitz condition is just replaced by the operator Lipschitz in $\mathcal{C}_{p}$, $1<p<\infty$ (one can construct a function $f$ on $[a, b]$ with positive derivative such that the inverse function $f^{-1}$ is not Lipschitz). However, the answer becomes positive if $f^{\prime}$ is assumed to be continuous.

(ii) The map considered in Problem 5 is norm closable. Indeed, if $A X_{n}-$ $X_{n} A \rightarrow_{n \rightarrow \infty} 0$ and $f(A) X_{n}-X_{n} f(A) \rightarrow_{n \rightarrow \infty} B$ then

$$
[B, A]=\lim _{n \rightarrow \infty}\left[\left[f(A), X_{n}\right], A\right]=\lim _{n \rightarrow \infty}\left[f(A),\left[X_{n}, A\right]\right]=0,
$$

that is, $B$ belongs to the commutant $\{A\}^{\prime}$ of $A$. If $\mathcal{E}: \mathcal{B}(H) \rightarrow\{A\}^{\prime}$ is a conditional expectation, then $B=\mathcal{E}(B)=\lim _{n \rightarrow \infty} \mathcal{E}\left(f(A) X_{n}-X_{n} f(A)\right)=$ $\lim _{n \rightarrow \infty}\left(f(A) \mathcal{E}\left(X_{n}\right)-\mathcal{E}\left(X_{n}\right) f(A)\right)=0$.

(iii) For the case $f(z)=\bar{z}$ the answer to Problem 5 is negative. More precisely the "Fuglede" map $A X-X A \rightarrow A^{*} X-X A^{*}$ is not $\mathrm{w}^{*}$-closable, if $\sigma(A)$ has non-empty interior and the spectral measure of $A$ is equivalent to the Lebesgue measure on the interior $U$ of $\sigma(A)$.

To see this, we assume for simplicity that $A$ is the operator of multiplication by $z$ on $L^{2}(U, d z d \bar{z})$. Let $f$ be the function on $G=\mathbb{R}^{2}$ given by $f(z)=\frac{\bar{z}}{z}$ and let $\varphi=N f$ be the corresponding Toeplitz multiplier on $G \times G$. It is not difficult to check that the set $E_{f}$ of all points $s \in G$ at which $f$ does not belong to $A(G)$ is the singleton $\{0\}$; applying Corollary 7.7 we get that $\kappa_{\varphi}^{w^{*}}=\Lambda=\{(z, z): z \in G\}$. It follows that the multiplier $\varphi$ is not $\mathrm{w}^{*}$-closable on $U \times U$, so there are Hilbert Schmidt operators $I_{h_{n}}$ supported in $U \times U$ with $I_{h_{n}} \rightarrow 0$ and $I_{\varphi h_{n}} \rightarrow B \neq 0$ in the weak* topology. We may assume that $h_{n}\left(z_{1}, z_{2}\right)$ vanish on some neighborhoods of the diagonal $\Lambda$. Indeed, let $V_{n}$ be a neighborhood of $\Lambda$ such that the Hilbert-Schmidt norm $\left\|h_{n} \chi_{V_{n}}\right\|_{2}$ is less than $1 / n$. Then $\left\|\varphi h_{n} \chi_{V_{n}}\right\|_{2}<1 / n$ whence $\left\|\varphi h_{n} \chi_{V_{n}}\right\|<1 / n$ and we may replace $h_{n}$ by $h_{n}-h_{n} \chi_{V_{n}}$.

Setting $p_{n}\left(z_{1}, z_{2}\right)=h_{n}\left(z_{1}, z_{2}\right) /\left(z_{1}-z_{2}\right)$ and $X_{n}=I_{p_{n}}$ we get that $\left[A, X_{n}\right]=I_{h_{n}} \rightarrow 0$ and $\left[A^{*}, X_{n}\right]=I_{\varphi h_{n}} \rightarrow B$.

\section{References}

[1] W.B. Arveson, Operator algebras and invariant subspaces, Ann. Math. (2) 100 (1974), 433-532 
[2] M.S. Birman and M.Z. Solomyak, Stieltjes double-integral operators. II, (Russian) Prob. Mat.Fiz. 2 (1967), 26-60

[3] M.S. Birman and M.Z. Solomyak, Stieltjes double-integral operators, III (Passage to the limit under the integral sign), (Russian) Prob. Mat. Fiz. No 6 (1973), $27-53$

[4] M.S. Birman and M.Z. Solomyak, Operator Integration, perturbations and commutators, Zap. Nauchn. Sem. Leningrad. Otdel. Mat. Inst. Steklov. (LOMI) Issled. Linein. Oper. Teorii Funktsii. 17, 170 (1989), 34-66

[5] M.S. Birman and M.Z. Solomyak, Double operator integrals in a Hilbert space, Int. Eq. Oper. Th. 47 (2003), no. 2, 131-168

[6] D.P. Blecher and R. Smith, The dual of the Haagerup tensor product, J. London Math. Soc. (2) 45 (1992), 126-144

[7] M. Bozejko and G. Fendler, Herz-Schur multipliers and completely bounded multipliers of the Fourier algebra of a locally compact group, Colloquium Math. 63 (1992) 311-313

[8] W.W. Comfort and H. Gordon, Vitali's theorem for invariant measures, Trans. Amer. Math. Soc. 991961 83-90

[9] J.L. Daletskii and S.G. Krein, Integration and differentiation of functions of hermitian operators and applications to the theory of perturbations, Amer. Math. Soc. Translations (2) 47 (1965), 1-30

[10] J.A. Erdos, A. Katavolos and V.S. Shulman, Rank one subspaces of Bimodules over Maximal Abelian Selfadjoint Algebras, J. Funct. Anal. 157 No.2 (1998), 554-587

[11] Yu.B. Farforovskaya, An estimate of the norm $\|f(A)-f(B)\|$ for selfadjoint operators $A$ and B, Zap. Nauchn. Semin. LOMI 56 (1976), 143-162 (English transl. J. Sov. Math. 14 (1980), 1133-1149)

[12] J. Froelich, Compact operators, invariant subspaces and spectral synthesis, J. Funct. Anal. 81 (1988), 1-37

[13] I. Gelfand, D. Raikov, G. Shilov, Commutative normed rings. Translated from the Russian, with a supplementary chapter, Chelsea Publishing Co., New York, 1964

[14] I.C. Gohberg and M.G. Krein, Theory and applications of Volterra operators in Hilbert space, Translation of Mathematical Monographs, vol. 24, American Mathematical Society, 1970

[15] C.Graham and O.C. McGehee Essays in commutative harmonic analysis, Springer-Verlag, New York-Berlin, 1979

[16] A. Grothendieck, Resume de la theorie metrique des produits tensoriels topologiques, Boll. Soc. Mat. Sao-Paulo 8 (1956), 1-79

[17] E. Hewitt and K.A. Ross, Abstract harmonic analysis. Vol. I. Structure of topological groups, integration theory, group representations, Springer-Verlag, Berlin-New York, 1979

[18] E. Hewitt and K. Stromberg, Real and abstract analysis. A modern treatment of the theory of functions of a real variable, Springer-Verlag, New York, 1965

[19] T. Kato, Perturbation theory for linear operators, Springer-Verlag, Berlin, 1995

[20] Y. Katznelson, An introduction to harmonic analysis Third edition, Cambridge University Press, Cambridge, 2004 
[21] E. Kissin and V.S. Shulman, Operator multipliers, Pacific J. Math. 227 (2006), no. $1,109-141$

[22] E. Kissin and V.S. Shulman Classes of operator-smooth functions. I. OperatorLipschitz functions. Proc. Edinb. Math. Soc. (2) 48 (2005), no. 1, 151-173

[23] E. Kissin, and V.S. Shulman, On the range inclusion of normal derivations: variations on a theme by Johnson, Williams and Fong. Proc. London Math. Soc. (3) 83 (2001), no. 1, 176-198

[24] B.K. Lahiri, On translations of sets in topological groups J. Indian Math. Soc. (N.S.) 39 (1975), 173-180

[25] P. Mattila, Geometry of sets and measures in Euclidean spaces. Fractals and rectifiability, Cambridge University Press, Cambridge, 1995

[26] V. Peller, Hankel operators in the theory of perturbations of unitary and selfadjoint operators. (Russian) Funktsional. Anal. i Prilozhen. 19 (1985), no. 2, 37-51, 96

[27] D. Potapov and F.Sukochev, Operator Lipschitz functions in Scahtten-von Neumann classes, Acta Math., to appear

[28] V.S. Shulman and L. Turowska, Operator synthesis. I. Synthetic sets, bilattices and tensor algebras, J. Funct. Anal. 209 (2004), 293-331

[29] V.S. Shulman and L. Turowska, Operator synthesis II: Individual synthesis and linear operator equations J. Reine Angew. Math. 590 (2006), 143-187

[30] N. Spronk and L. Turowska, Spectral synthesis and operator synthesis for compact groups, J. London Math. Soc. (2) 66 (2002), 361-376

[31] W. Rudin, Fourier analysis on groups, John Wiley \& Sons, Inc., New York, 1990

\section{V.S. Shulman}

Department of Mathematics, Vologda State Technical University, Vologda, Russia e-mail: shulman_v@yahoo.com

\section{I.G. Todorov}

Department of Pure Mathematics, Queen's University Belfast, Belfast BT7 1NN, United Kingdom

e-mail: i.todorov@qub.ac.uk

L. Turowska

Department of Mathematical Sciences, Chalmers University of Technology and the University of Gothenburg, Gothenburg SE-412 96, Sweden

e-mail: turowska@chalmers.se 